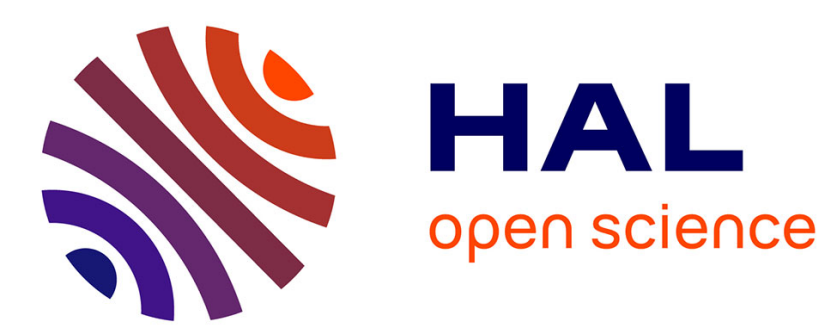

\title{
Optimal Torus Exploration by Oblivious Robots
}

\author{
Stéphane Devismes, Anissa Lamani, Franck Petit, Sébastien Tixeuil
}

\section{To cite this version:}

Stéphane Devismes, Anissa Lamani, Franck Petit, Sébastien Tixeuil. Optimal Torus Exploration by Oblivious Robots. NETYS 2015 - Third International Conference on Networked Systems, May 2015, Agadir, Morocco. pp.183-199, 10.1007/978-3-319-26850-7_13 . hal-00926573v3

\section{HAL Id: hal-00926573 https://inria.hal.science/hal-00926573v3}

Submitted on 13 Jun 2014

HAL is a multi-disciplinary open access archive for the deposit and dissemination of scientific research documents, whether they are published or not. The documents may come from teaching and research institutions in France or abroad, or from public or private research centers.
L'archive ouverte pluridisciplinaire HAL, est destinée au dépôt et à la diffusion de documents scientifiques de niveau recherche, publiés ou non, émanant des établissements d'enseignement et de recherche français ou étrangers, des laboratoires publics ou privés. 


\title{
Optimal Torus Exploration by Oblivious Mobile Robots
}

\author{
S. Devismes ${ }^{1}$, A. Lamani ${ }^{2}$, F. Petit ${ }^{3}$, and S. Tixeuil ${ }^{3}$ \\ ${ }^{1}$ VERIMAG UMR 5104, Université Joseph Fourier, France \\ ${ }^{2}$ MIS, Université de Picardie Jules Verne (France) \\ ${ }^{3}$ LIP6, UPMC Sorbonne Universités (France)
}

\begin{abstract}
We consider autonomous robots that are endowed with motion actuators and visibility sensors. The robots we consider are weak, i.e., they are anonymous, uniform, unable to explicitly communicate, and oblivious (they do not remember any of their past actions). In this paper, we propose an optimal (w.r.t. the number of robots) solution for the terminating exploration of a torus-shaped network by a team of $k$ such robots. In more details, we first show that it is impossible to explore a simple torus of arbitrary size with (strictly) less than four robots, even if the algorithm is probabilistic. If the algorithm is required to be deterministic, four robots are also insufficient. This negative result implies that the only way to obtain an optimal algorithm (w.r.t. the number of robots participating to the algorithm) is to make use of probabilities. Then, we propose a probabilistic algorithm that uses four robots to explore all simple tori of size $\ell \times L$, where $7 \leq \ell \leq L$. Hence, in such tori, four robots are necessary and sufficient to solve the (probabilistic) terminating exploration. As a torus can be seen as a 2-dimensional ring, our result shows, perhaps surprisingly, that increasing the number of possible symmetries in the network (due to increasing dimensions) does not come at an extra cost w.r.t. the number of robots that are necessary to solve the problem.
\end{abstract}

Keywords: Robot, Torus, Exploration, Obliviousness

\section{Introduction}

We consider autonomous robots that are endowed with motion actuators and visibility sensors, but that are otherwise unable to communicate. They evolve in a discrete environment, where the space is partitioned into a finite number of locations, conveniently represented by a graph, where the nodes represent the possible locations that a robot can take and the edges the possibility for a robot to move from one location to another. Those robots must collaborate to solve a collective task despite being limited with respect to inputs from the environment, asymmetry, memory, etc. In particular, the robots we consider are anonymous, uniform, yet they can sense their environment and take decisions according to their own ego-centered view. In addition, they are oblivious, i.e., they do not remember their past actions. The three execution models are denoted (using recent taxonomy [1]) FSYNC, for fully synchronous, SSYNC, for semi-synchronous, and ASYNC, for asynchronous. In the ASYNC model, robots execute their phases in a completely independent manner. In the SSYNC model an arbitrary non-empty subset of robots execute the three phases synchronously and atomically. In the FSYNC model all robots execute the three phases synchronously.

In this context, typical problems are terminating exploration $[2,3,4,5,6]$, exclusive perpetual exploration [7, 8, 9], exclusive searching [10,9], and gathering [9, 11, 12]. Here, we study the terminating exploration (or simply exploration) problem, which requires that robots collectively explore the whole graph and stop upon completion. We focus on the case when the network is an anonymous unoriented torus. Since 
the robots are unable to communicate explicitly and have no persistent memory, they are unable to remember the various steps taken before. Therefore, the positions of the other robots are the only way to distinguish the different stages of the exploration process. The main complexity measure is then the minimum number of robots that are required to achieve exploration. Since numerous symmetric configurations induce a large number of required robots, minimizing the number of robots turns out to be a difficult problem.

Related Work. With respect to the (terminating) exploration problem, minimizing the number of robots for exploring particular classes of graphs led to contrasted results. The only result available for exploration in general graphs [2] considers that edges are labeled in such a way that the network configuration (made of the topology, the edge labeling, and the robot positions) is asymmetric. In this extended model, three robots are not sufficient to explore all asymmetric configurations, and four robots are sufficient to explore all asymmetric configurations. Note that exploring the set of asymmetric configurations is strictly weaker than exploring the complete underlying graph, especially when the graph is highly symmetric. The rest of the literature is thus dedicated to a weaker model, where edges are not labeled (or equivalently, the labeling is decided by an adversary anytime a robot is activated). One extreme case in this weak model is the set of tree-shaped networks, as in general, $\Omega(n)$ robots are necessary and sufficient to explore a tree network of $n$ nodes deterministically [5]. The other extreme case is the set of grid-shaped networks [3], where three robots are necessary and sufficient to explore deterministically any grid of at least three nodes (except for the grids of size $2 \times 2$ and $3 \times 3$, where four - respectively five - robots are necessary and sufficient). However, this result is mainly due to the fact that grids are not regular graphs: they contain nodes of degrees 2, 3, and 4. This topological property implies less symmetries. In contrast, rings and tori are regular graphs, and consequently more intricate. In ring-shaped networks [6], the fact that the number $k$ of robots and the ring size $n$ must be coprime yields a $\Omega(\log n)$ lower bound, since the smallest non-divisor of $n$ evolves as $\log n$ in the worst case. The case where $k$ and $n$ are coprime has been studied in the deterministic setting [13], and five asynchronous robots are necessary and sufficient in this case. The large number of robots induced by the deterministic setting in ring-shaped networks hinted at a possible more efficient solution when robots can make use of probabilities [4]. As a matter of fact, four robots are necessary and sufficient to probabilistically explore any ring of size at least four. While the gain in going probabilistic is only one robot when $k$ and $n$ are coprime, a logarithmic factor is obtained in all remaining cases. Aforementioned deterministic solutions typically operate in the ASYNC model, while probabilistic ones can only cope with the weaker SSYNC model. Actually, an impossibility result [4] explicitly states that randomization does not help in the ASYNC model (that is, there exists a scheduling such that random choices are all nullified).

So far, no research explored the feasibility of exploring a torus-shaped network with a team of $k$ robots. The theoretical interest of such a setting lies in the additional axes of symmetry (w.r.t. ring shaped networks). As previously stated, the exploration (with stop) process is intrinsically related to the ability to differentiate consecutive phases of the exploration. More possible symmetries hint that more robots than in rings are required to complete exploration: As robots have no way to distinguish and to agree on some kind of orientation, e.g., North-South/Est-West, somehow the current robot configuration has to encode consistent information for both axes.

Contribution. We propose an optimal (w.r.t. the number of robots) solution for the terminating exploration of a torus-shaped network by a team of $k$ such robots. In more details, we first show that it is impossible to explore a simple torus of arbitrary size with less than four robots, even if the algorithm is probabilistic. If the algorithm is required to be deterministic, four robots are also insufficient. This negative result implies that the only way to obtain an optimal algorithm (w.r.t. the number of robots participating to the algorithm) is to make use of probabilities, and thus, within SSYNC, due to aforementioned impossibilities [4].

So, we propose a probabilistic algorithm designed for the SSYNC model that uses four robots to explore 
all simple tori of size $\ell \times L$, where $7 \leq \ell \leq L$. Hence, in such tori, four robots are necessary and sufficient to solve the (probabilistic) terminating exploration. As a torus can be seen as a 2-dimensional ring, our result shows, perhaps surprisingly, that increasing the number of possible symmetries in the network (due to increasing dimensions) does not bring an extra cost with respect to the number of robots that are necessary to solve the problem.

Roadmap. Section 2 presents the system model and the problem to be solved. Lower bounds are shown in Section 3. The general solution using four robots is given in Section 4. The correctness of this solution is given in Section 5. Section 6 gives some concluding remarks.

\section{Preliminaries}

Distributed Systems. We consider systems of autonomous mobile entities called robots evolving in a simple unoriented connected graph $G=(V, E)$, where $V$ is a finite set of $n$ nodes (i.e., $|V|=n)$ and $E$ a finite set of edges, i.e., unordered pairs of distinct nodes. Nodes represent locations that robots can take and edges represent the possibility for a robot to move from one location to another. Two nodes $u$ and $v$ are neighbors in $G$ if and only if $\{u, v\} \in E$.

We assume that $G$ is an $(\ell, L)$-Torus (or a Torus, for short), where $\ell$ and $L$ are two positive integers, i.e., $G$ satisfies the following two conditions: $(i) n=\ell \times L$ and ( $i i)$ there exists an order $v_{1}, \ldots, v_{n}$ on the nodes of $V$ such that $\forall i \in[1 . . n]$ :

- If $i+\ell \leq n$, then $\{i, i+\ell\} \in E$, else $\{i,(i+\ell) \bmod n\} \in E$.

- If $i \bmod \ell \neq 0$, then $\{i, i+1\} \in E$, else $\{i, i-\ell+1\} \in E$.

Given the previous order $v_{1}, \ldots, v_{n}$, for every $j \in[0 . .(L-1)]$, the sequence $v_{1+j \times \ell}, v_{2+j \times \ell}, \ldots, v_{\ell+j \times \ell}$ is called an $\ell$-ring. Similarly, for every $k \in[1 . . \ell], v_{k}, v_{k+\ell}, v_{k+2 \times \ell}, \ldots, v_{k+(L-1) \times \ell}$ is called an $L$-ring. Note that when $\ell=L$, any $\ell$-ring is also an $L$-ring and conversely. More generally, we use the term ring to arbitrarily designate an $\ell$-ring or an $L$-ring.

Remark 1 As an $(\ell, L)$-torus is a simple graph, every node has four distinct neighbors, and consequently we have: $3 \leq \ell \leq L$ and $n=\ell \times L \geq 9$.

Nodes are assumed to be anonymous (they have no access to identifiers or other symmetry breaking capabilities). Moreover, given two neighboring nodes $u$ and $v$, we assume that there is no explicit or implicit labeling allowing the robots to determine whether $u$ is either on the left, on the right, above, or below $v$. However, for the purpose of proof description, we may use indices for nodes or robots.

An isomorphism of graphs $G$ and $H$ is a bijection $f$ between the vertex sets of $G$ and $H$ such that any two nodes $u$ and $v$ of $G$ are neighbors in $G$ if and only if $f(u)$ and $f(v)$ are neighbors in $H$. When $G$ and $H$ are one and the same graph, $f$ is called an automorphism of $G$. Remark that an $(\ell, L)$-Torus and an $(L, \ell)$-Torus are isomorphic. Hence, as the nodes are anonymous, an $(\ell, L)$-Torus cannot be distinguished from an $(L, \ell)$-Torus. So, without loss of generality, we always consider $(\ell, L)$-Tori, where $\ell \leq L$.

Robots and Computation. Operating on $G$ are $k$ robots. The robots do not communicate in an explicit way; however they see the position of all other robots in their ego-centered coordinate system and can acquire knowledge from this information.

Each robot operates according to its (local) program. We call protocol a collection of $k$ programs, each one operating on a single robot. Here we assume that robots are uniform and anonymous, i.e., they all have the same program using no parameter allowing to differentiate them. 
The program of a robot consists in executing Look-Compute-Move cycles infinitely many times. That is, the robot first observes its environment (Look phase). Based on its observation, a robot then (probabilistically or deterministically) decides to move or stay idle (Compute phase). When a robot decides to move, it moves toward its destination during the Move phase.

During the Compute phase, the decision between moving or staying idle is either deterministic or probabilistic. In the latter case, the robot decides between moving and staying idle using some fixed probability $p$, with $0<p<1$; and we say that the robot tries to move.

We assume that robots cannot remember any previous observation nor computation performed in any previous cycle. Such robots are called oblivious.

We consider the semi-synchronous model [14]. In this model, time is represented by an infinite sequence of instants $0,1,2, \ldots$ No robot has access to this global time. At each instant, a non-empty subset of robots is activated. Every robot that is activated at instant $t$ atomically executes a full cycle between $t$ and $t+1$. Activations are determined by an adversary.

Note that in this model, any robot performing a Look operation sees all other robots on nodes and not on edges.

Multiplicity. We assume that during the Look phase, every robot can perceive whether several robots are located on the same node. This ability is called (global) multiplicity detection. We shall indicate by $d_{i}(t)$ the multiplicity of robots present in node $v_{i}$ at instant $t$. We consider two versions of multiplicity detection: the strong and weak multiplicity detections. Under the weak multiplicity detection, for every node $v_{i}, d_{i}$ is a function $\mathbb{N} \mapsto\{\circ, \perp, \top\}$ defined as follows: $d_{i}(t)$ is equal to either $\circ, \perp$, or $\top$ according to $v_{i}$ contains none, one or several robots at instant $t$. If $d_{i}(t)=0$, then we say that $v_{i}$ is free at instant $t$, otherwise $v_{i}$ is occupied at instant $t$. If $d_{i}(t)=\top$, then we say that $v_{i}$ contains a tower at instant $t$. Under the strong multiplicity detection, for every node $v_{i}, d_{i}$ is a function $\mathbb{N} \mapsto \mathbb{N}$ where $d_{i}(t)=x$ indicates that there are $x$ robots in node $v_{i}$ at instant $t$. If $d_{i}(t)=0$, then we say that $v_{i}$ is free at instant $t$, otherwise $v_{i}$ is occupied at instant $t$. If $d_{i}(t)>1$, then we say that $v_{i}$ contains a tower (of $d_{i}(t)$ robots) at instant $t$.

Configurations, Views and Executions. To define the notion of configuration, we need to use an arbitrary order $\prec$ on nodes. The system being anonymous, robots do not know this order. Let $v_{1}, \ldots, v_{n}$ be the list of the nodes in $G$ ordered by $\prec$. The configuration at instant $t$ is $d_{1}(t), \ldots, d_{n}(t)$. We denote by initial configurations the configurations from which the system can start at instant 0 . Every configuration from which no robot moves or tries to move if activated is said to be terminal.

The view of robot $\mathcal{R}$ at instant $t$ is a labeled graph isomorphic to $G$, where every node $v_{i}$ is labeled by $d_{i}(t)$, except the node where $\mathcal{R}$ is currently located, this latter node $v_{j}$ is labeled by $d_{j}(t), *$. (Indeed, the coordinate system is ego-centered.) Hence, from its view, a robot can compute the view of each other robot, and decide whether some other robots have the same view as its own. The views $\mathcal{V}$ and $\mathcal{V}^{\prime}$ are identical if and only if there exists an isomorphism $f$ of $\mathcal{V}$ and $\mathcal{V}^{\prime}$ such that every node $v$ of $\mathcal{V}$ has the same label in $\mathcal{V}$ as node $f(v)$ in $\mathcal{V}^{\prime}$. For example, in Figure 1 , the robots located at node $v_{1}$ and $v_{5}$ respectively have identical views: to see this, apply the isomorphism $f$ such that $f\left(v_{1}\right)=v_{5}, f\left(v_{2}\right)=v_{2}, f\left(v_{3}\right)=v_{8}, f\left(v_{4}\right)=v_{4}$, $f\left(v_{5}\right)=v_{1}, f\left(v_{6}\right)=v_{7}, f\left(v_{7}\right)=v_{6}, f\left(v_{8}\right)=v_{3}$, and $f\left(v_{9}\right)=v_{9}$ on the view of the node located at $v_{1}$. Conversely, the view of the node located at $v_{2}$ is different of the two others. 


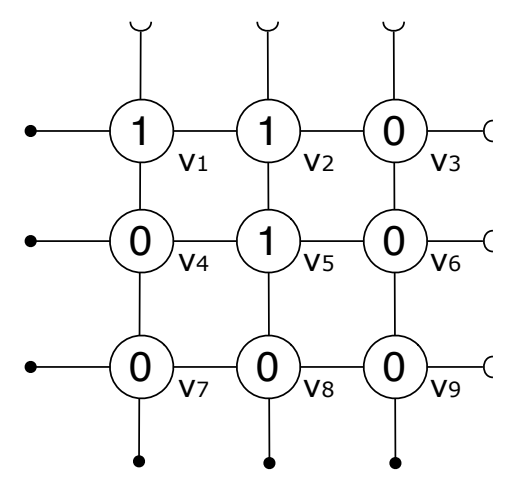

Figure 1: Configuration where two robots have identical views. Numbers inside nodes indicate the strong multiplicity value.

Every decision to move is based on the view obtained during the last Look action. However, it may happen that some edges incident to a node $v$ currently occupied by the deciding robot look identical in its view, i.e., $v$ lies on a symmetric axis of its view. In this case, if the robot decides to take one of these edges, it may take any of them. We assume the worst-case decision in such cases, i.e., the actual edge among the identically looking ones is chosen by the adversary.

Two configurations $d_{1}, \ldots, d_{n}$ and $d_{1}^{\prime}, \ldots, d_{n}^{\prime}$ are indistinguishable (resp., distinguishable otherwise) if and only if there exists an automorphism on $G, f: V \mapsto V$ such that $\forall i \in\{1, \ldots, n\}, d_{i}=d_{j}^{\prime}$ where $v_{j}=f\left(v_{i}\right)$. For example, Configurations $(a)$ and $(b)$ in Figure 2 are indistinguishable: to see this, apply the automorphism $f$ such that $f\left(v_{1}\right)=v_{2}, f\left(v_{2}\right)=v_{5}, f\left(v_{3}\right)=v_{8}, f\left(v_{4}\right)=v_{3}, f\left(v_{5}\right)=v_{6}, f\left(v_{6}\right)=v_{9}$, $f\left(v_{7}\right)=v_{1}, f\left(v_{8}\right)=v_{4}$, and $f\left(v_{9}\right)=v_{7}$. Conversely, configuration $(c)$ is distinguishable from (a) (resp., $(b))$.

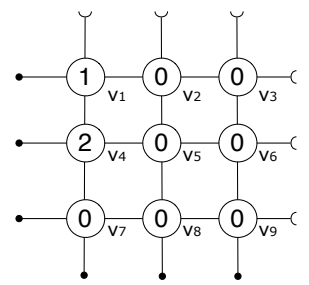

(a)

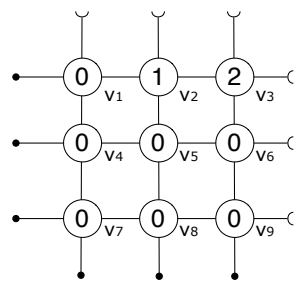

(b)

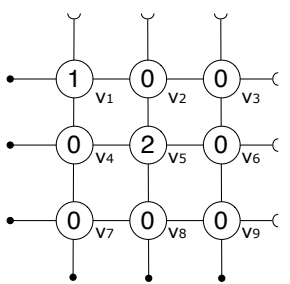

(c)

Figure 2: Example of indistinguishable and distinguishable configurations in a (3,3)-torus. Numbers inside nodes indicate the strong multiplicity value.

Consider two indistinguishable configurations $\gamma$ and $\gamma^{\prime}$. Assume that some robot $\mathcal{R}$ is located at node $v_{i}$ in $\gamma$. Then, by definition, there is some robot $\mathcal{R}^{\prime}$ located at node $f\left(v_{i}\right)$ in $\gamma^{\prime}$. Moreover, the view of $\mathcal{R}$ in $\gamma$ is the same as the view of $\mathcal{R}^{\prime}$ in $\gamma^{\prime}$. So, if $\mathcal{R}$ is activated in $\gamma$ and may decide to move to some neighboring node $v_{j}$, then it is the same for $\mathcal{R}^{\prime}$ : if $\mathcal{R}^{\prime}$ is activated in $\gamma^{\prime}$, it may decide to move to $f\left(v_{j}\right)$. For example, assume that the robot located at $v_{1}$ in Configuration $(a)$ of Figure 2 is activated and decides to move toward $v_{4}$. Then, there is a possible step where the robot located at node $v_{2}=f\left(v_{1}\right)$ in Configuration $(b)$ moves toward $v_{3}=f\left(v_{4}\right)$.

A scheduling is a list of activation's choices that can be made by the adversary, i.e., a scheduling is any infinite list of non-empty subset of robots $\sigma_{0}, \sigma_{1}, \ldots$, where $\forall i \geq 0, \sigma_{i}$ is the set of robots activated at instant $i$. An infinite list of configurations $\gamma_{0}, \gamma_{1}, \ldots$ can be generated from the scheduling $\sigma_{0}, \sigma_{1}, \ldots$ if and only if $\forall i \geq 0, \gamma_{i+1}$ can be obtained from $\gamma_{i}$ after each robot in $\sigma_{i}$ is activated at instant $i$ to atomically perform a cycle (in this case, $\gamma_{i} \gamma_{i+1}$ is step). 
We call execution any infinite list of configurations $\gamma_{0}, \gamma_{1}, \ldots$ that can be generated from an arbitrary scheduling and such that $\gamma_{0}$ is a possible initial configuration. An execution $e$ terminates if $e$ contains a terminal configuration.

We restrict the power of the adversary by assuming that schedulings are fair: a scheduling $\sigma_{0}, \sigma_{1}, \ldots$ is fair if and only if for every robot $\mathcal{R}$, for every instant $i$, there exists an instant $j \geq i$ such that $\mathcal{R} \in \sigma_{j}$. An execution $e$ is fair if and only if $e$ can be generated by a fair scheduling.

A particular case of fair scheduling is the sequential fair scheduling: a scheduling $\sigma_{0}, \sigma_{1}, \ldots$ that is fair and such that $\forall i \geq 0,\left|\sigma_{i}\right|=1$. An execution $e$ is sequential fair if it can be generated from a sequential fair scheduling.

Exploration. We consider the exploration problem, where $k$ robots, initially placed at different nodes of a graph $G=(V, E)$, collectively explore $G$ before stopping moving forever. By "collectively" we mean that every node of $G$ is eventually visited by at least one robot. More formally, we say that a protocol $\mathcal{P}$ deterministically (resp., probabilistically) solves the exploration problem assuming a fair scheduling if and only if every fair execution $e$ of $\mathcal{P}$ starting from a towerless configuration satisfies: (1) $e$ reaches a terminal configuration in finite time (resp., with probability one ${ }^{1}$ ), and (2) every node is visited by at least one robot during $e$. Note that the previous definition implies that every initial configuration are towerless. Note also that in case of probabilistic exploration, termination is not certain, however the overall probability of non-terminating executions is 0 . Observe that the exploration problem is not defined for $k>n$ and is straightforward for $k=n$. (In this latter case, the exploration is already accomplished in the initial towerless configuration.)

\section{Lower bound}

In this section, we first generalize to arbitrary topologies a result from [4], that was given for rings. We then instantiate this result to obtain a lower bound (actually 4) on the number of robots required to solve (deterministic or probabilistic) exploration in any torus.

To be as general as possible, we assume here that the multiplicity detection of robots is strong. Moreover, we consider any (deterministic or probabilistic) exploration protocol $\mathcal{P}$ using a team of $k$ robots in an arbitrary topology $G=(V, E)$ of $n$ nodes (i.e., $n=|V|)$.

First, assume that $n>k$. Then, the exploration is not (trivially) accomplished in an initial configuration. As robots are oblivious, any terminal configuration of $\mathcal{P}$ in that case should be different from any possible initial configuration. Now, the set of possible initial configurations is exactly the set of all towerless configurations. So, any terminal configuration necessarily contains a tower:

Remark 2 If $n>k$, any terminal configuration of $\mathcal{P}$ contains at least one tower.

Consider an instant $t$. Despite a non-empty subset of robots being activated at instant $t$, it is possible that no robot moves between $t$ and $t+1$. Such "empty" steps being useless, we may consider the minimal relevant subsequence of the execution defined below, where all "empty" steps have been removed:

Definition 1 (MRS) Let $s$ be a sequence of configurations. The minimal relevant subsequence of $s$, noted $\mathcal{M R S}(s)$, is the maximal subsequence of $s$ where no two consecutive configurations are identical.

\footnotetext{
${ }^{1}$ We recall that "with probability one" has the same meaning as "almost surely" or "almost certainly," but is different of "asymptotically almost surely," see [15], page 186.
} 
Lemma 1 Assume that $n>k$. Let e be any sequential fair execution e of $\mathcal{P}$ that terminates, ${ }^{2} \mathcal{M R S}(e)$ has at least $n-k+1$ configurations containing a tower of less than $k$ robots.

Proof. Assume, by the contradiction, that there is a sequential fair execution $e$ of $\mathcal{P}$ that terminates and such that $\mathcal{M R S}(e)$ has less than $n-k+1$ configurations containing a tower of less than $k$ robots.

Take the last configuration $\alpha$ without tower that appears in $e$ and all remaining configurations that follow in $e$ (all of them contains a tower) and form $e^{\prime}$ ( $e^{\prime}$ necessarily exists, by Remark 2). As $\alpha$ could be an initial configuration and $e$ is a sequential fair execution that terminates, $e^{\prime}$ is also a sequential fair execution of $\mathcal{P}$ that terminates.

By definition, $\mathcal{M R S}\left(e^{\prime}\right)$ is constituted of a configuration with no tower only followed by configurations with tower and $n-k$ new nodes (remember that $k$ nodes are already visited in the initial configuration) must be visited before $e^{\prime}$ reaches its terminal configuration.

Consider a step $\beta \beta^{\prime}$ in $e^{\prime}$.

1. If $\beta=\beta^{\prime}$, then no node is visited during the step.

2. If $\beta \neq \beta^{\prime}$, then there are three possible cases:

(a) $\beta$ contains no towers. In this case, $\beta=\alpha$ (the initial configuration of $e^{\prime}$ ) and $\beta^{\prime}$ contains a tower. As only one robot moves in $\beta \beta^{\prime}$ to create a tower ( $e^{\prime}$ is sequential), no node is visited during this step.

(b) $\beta$ contains a tower and $\beta^{\prime}$ contains a tower of $k$ robots. As $e^{\prime}$ is sequential and all robots are located at the same node in $\beta^{\prime}$, one robot moves to an already occupied node in $\beta \beta^{\prime}$ and no node is visited during this step.

(c) $\beta$ contains a tower and $\beta^{\prime}$ contains a tower of less than $k$ robots. In this case, at most one node is visited in $\beta \beta^{\prime}$ because $e^{\prime}$ is sequential.

To sum up, only the steps from a configuration containing a tower to a configuration containing a tower of less than $k$ robots (Case 2.(c)) allow to visit at most one node each time. Now, in $\mathcal{M R \mathcal { S }}\left(e^{\prime}\right)$ there are less than $n-k+1$ configurations containing a tower of less than $k$ robots and the first of these configurations appearing into $e^{\prime}$ is consecutive to a step starting from the initial configuration (Case 2.(a)). Hence, less than $n-k$ nodes are dynamically visited during $e^{\prime}$ and, as exactly $k$ nodes are visited in the initial configuration, less than $n$ nodes are visited when $e^{\prime}$ terminates, a contradiction.

Lemma 2 If $n>k$, then for every sequential fair execution e of $\mathcal{P}$ that terminates, $\mathcal{M R S}(e)$ has at least $n-k+1$ configurations containing a tower of less than $k$ robots and any two of them are distinguishable.

Proof. Consider any sequential fair execution $e=\gamma_{0}, \gamma_{1}, \ldots$ of $\mathcal{P}$ that terminates. As $e$ terminates, $\mathcal{M R S}(e)$ has a finite number of configurations. So, let $x$ be the number of configurations in $\mathcal{M R S}(e)$ containing a tower of less than $k$ robots. By Lemma $1, x \geq n-k+1$.

We first show that $\left(^{*}\right)$ if $\mathcal{M R S}(e)$ contains at least two configurations having a tower of less than $k$ robots that are indistinguishable, then there exists a sequential fair execution $e^{\prime}$ that terminates and such that $\mathcal{M R S}\left(e^{\prime}\right)$ has $x^{\prime}$ configurations containing a tower of less than $k$ robots, where $x^{\prime}<x$.

Assume that there are two indistinguishable configurations $\gamma_{t}=d_{1}^{t}, \ldots, d_{n}^{t}$ and $\gamma_{t^{\prime}}=d_{1}^{t^{\prime}}, \ldots, d_{n}^{t^{\prime}}$ in $\mathcal{M R S}(e)$ having a tower of less than $k$ robots. Without loss of generality, assume that $t^{\prime}>t$. By definition, there exists an automorphism $f$ on $G$ such that $\forall i \in\{1, \ldots, n\}, d_{i}^{t}=d_{j}^{t^{\prime}}$ where $v_{j}=f\left(v_{i}\right)$. Then,

\footnotetext{
${ }^{2}$ As explained in the model, in case where $\mathcal{P}$ is probabilistic, some of its executions may not terminate, even if the overall probability that such executions occur is 0 .
} 
$e^{\prime}=\gamma_{0}, \ldots, \gamma_{t}, \gamma_{t^{\prime}+1}^{\prime}, \gamma_{t^{\prime}+2}^{\prime}, \ldots$ is a sequential fair execution of $\mathcal{P}$, where that $\forall z \geq t^{\prime}+1$, we have $\gamma_{z}^{\prime}=d_{g(1)}^{z}, \ldots, d_{g(n)}^{z}$ where $g$ is a bijection such that $\forall s \in[1 . . n], f\left(v_{s}\right)=v_{g(s)}$ and $\gamma_{z}=d_{1}^{z}, \ldots, d_{n}^{z}$. Moreover, $\mathcal{M R S}\left(e^{\prime}\right)$ has $x^{\prime}$ configurations containing a tower of less than $k$ robots, where $x^{\prime}<x$.

By (*), if $\mathcal{M R S}(z)$ contains less than $n-k+1$ distinguishable configurations altogether with a tower of less than $k$ robots, it is possible to (recursively) construct a sequential fair execution $e^{\prime}$ of $\mathcal{P}$ that terminates such that $\mathcal{M R S}\left(e^{\prime}\right)$ has less than $n-k+1$ configurations containing a tower of less than $k$ robots, a contradiction to Lemma 1 . Hence, the lemma holds.

From Lemma 2, we can deduce the following corollary:

Corollary 1 Considering any (probabilistic or deterministic) exploration protocol for $k$ robots on a graph of $n>k$ nodes working under any fair scheduling, there exists a set $\mathcal{S}$ of at least $n-k+1$ configurations such that:

1. any two different configurations in $\mathcal{S}$ are distinguishable, and

2. in every configuration in $\mathcal{S}$, there is a tower of less than $k$ robots.

Theorem 1 Assuming fair schedulings, $\forall k, 0 \leq k<3$, there is no protocol to (deterministically or probabilistically) explore any torus of $n$ nodes using $k$ robots.

Proof. First, $k<9 \leq n=\ell \times L$ (see Remark 1). Then, for $k=0$, the theorem is trivially verified. Consider now the case $k=1$ and $k=2$. With one robot it is impossible to construct a configuration with a tower; and with two robots it is impossible to construct a configuration with a tower of less than $k$ robots ( $k=2$ ). Hence, for $k=1$ and $k=2$, the theorem is a direct consequence of Corollary 1 .

The previous theorem excludes that $\mathcal{P}$ works with $k<3$. Let now assume that $k=3$ and consider any arbitrary $(\ell, L)$-torus (remember that $n=\ell \times L \geq 9$, Remark 1 ). Then, by Corollary 1, we should be able to exhibit a set $\mathcal{S}$ of $n-2$ configurations such that:

1. Any two different configurations in $\mathcal{S}$ are distinguishable, and

2. In every configuration in $\mathcal{S}$, there is a tower of 2 robots.

Such configurations differ according to the relative positions of the tower and the robot which is alone. Two cases are then possible depending on whether $\ell=L$ or $\ell<L$. In the former case, the size of $\mathcal{S}$ is bounded by $\sum_{i=2}^{\left\lfloor\frac{L}{2}\right\rfloor+1} i=\frac{\left\lfloor\frac{L}{2}\right\rfloor \times\left(\left\lfloor\frac{L}{2}\right\rfloor+3\right)}{2}$. For example (refer to Figure 3), in a $(5,5)$-torus, the size of $\mathcal{S}$ is at most 5 . 


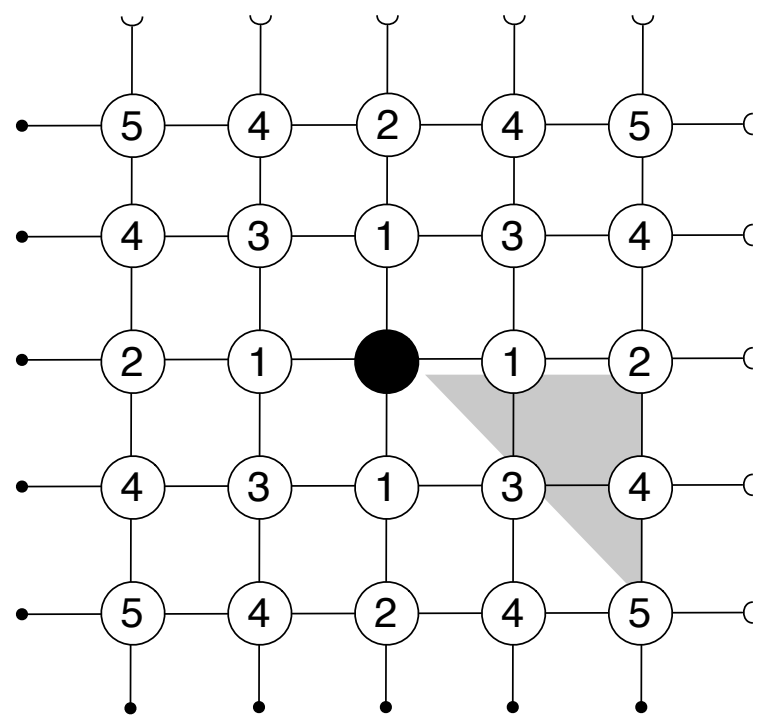

Figure 3: For every value $i$ inside a white node, every two configurations where (1) the black node contains the tower of two robots and (2) any white node of number $i$ contains the single robot are indistinguishable.

In the latter case, the size of $\mathcal{S}$ is bounded by $\left(\left\lfloor\frac{\ell}{2}\right\rfloor+1\right)\left(\left\lfloor\frac{L}{2}\right\rfloor+1\right)-1$. For example (refer to Figure 4), in a $(5,6)$-torus, the size of $\mathcal{S}$ is at most 11.

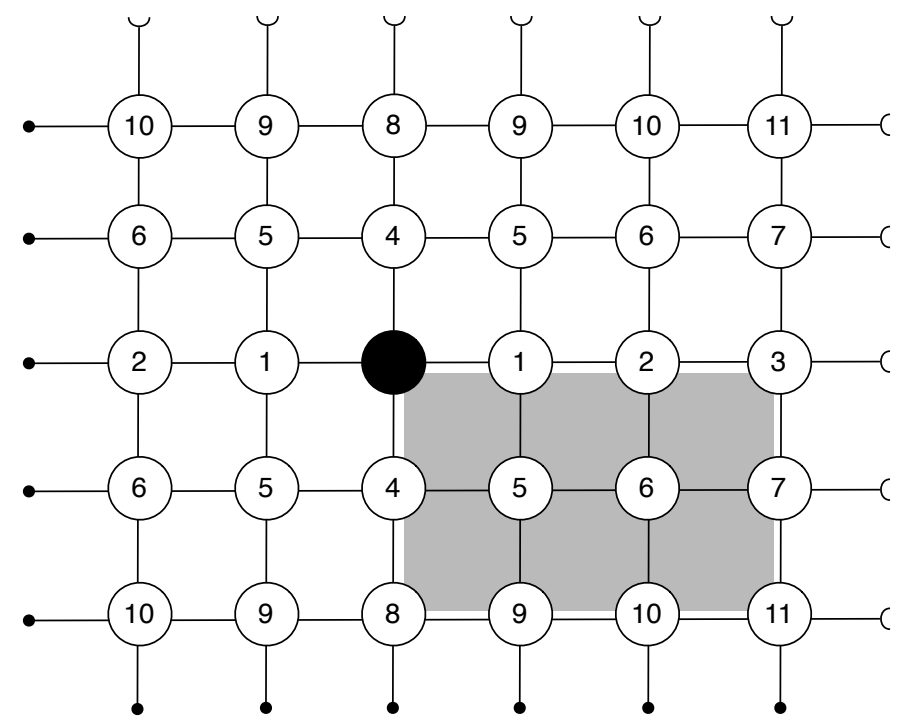

Figure 4: For every value $i$ inside a white node, every two configurations where (1) the black node contains the tower of two robots and (2) any white node of number $i$ contains the single robot are indistinguishable.

Let now study the case where $\ell=L$. Then, $\frac{\left\lfloor\frac{L}{2}\right\rfloor \times\left(\left\lfloor\frac{L}{2}\right\rfloor+3\right)}{2}$ should be greater or equal to $n-2$, i.e., $L^{2}-2$. 


$$
\begin{array}{ll}
\frac{\left\lfloor\frac{L}{2}\right\rfloor \times\left(\left\lfloor\frac{L}{2}\right\rfloor+3\right)}{2} & \geq L^{2}-2 \\
\left\lfloor\frac{L}{2}\right\rfloor \times\left(\left\lfloor\frac{L}{2}\right\rfloor+3\right) & \geq 2 L^{2}-4 \\
\frac{L^{2}}{4}+\frac{3 L}{2} & \geq 2 L^{2}-4 \\
\frac{L^{2}+6 L}{4} & \geq 2 L^{2}-4 \\
L^{2}+6 L & \geq 8 L^{2}-16 \\
7 L^{2}-6 L-16 & \leq 0
\end{array}
$$

$\Delta=484>0$, so $7 L^{2}-6 L-16=0$ has two solutions: $\frac{6-\sqrt{484}}{14}$ and $\frac{6+\sqrt{484}}{14}$; and $7 L^{2}-6 L-16 \leq 0$ for $L \in\left[\frac{6-\sqrt{484}}{14} ; \frac{6+\sqrt{484}}{14}\right]$. Now $L \geq 3$ (Remark 1 ) and $\frac{6+\sqrt{484}}{14}=2$. So, we obtain a contradiction: there is neither probabilistic nor deterministic exploration protocol in that case, even assuming a fair scheduling.

Let now study the case where $\ell<L$. Then, $\left(\left\lfloor\frac{\ell}{2}\right\rfloor+1\right)\left(\left\lfloor\frac{L}{2}\right\rfloor+1\right)-1$ should be greater or equal to $n-2$, i.e., $\ell \times L-2$.

$$
\begin{aligned}
& \left(\left\lfloor\frac{\ell}{2}\right\rfloor+1\right)\left(\left\lfloor\frac{L}{2}\right\rfloor+1\right)-1 \geq \ell \times L-2 \\
& \frac{\ell \times L}{4}+\frac{\ell}{2}+\frac{L}{2}+1 \geq \ell \times L-1 \\
& \frac{\ell \times L+2 \ell+2 L}{4} \geq \ell \times L-2 \\
& \ell \times L+2 \ell+2 L \geq 4 \ell \times L-8 \\
& 2 \ell+2 L+8 \geq 3 \ell \times L
\end{aligned}
$$

Now, as $3 \leq \ell<L$ (Remark 1), $2 \ell+2 L+8 \geq 3 \ell \times L$ has no solution: there is neither probabilistic nor deterministic exploration protocol in that case, even assuming a fair scheduling.

Hence, there is neither probabilistic nor deterministic protocol to explore any torus with 3 robots and, with Theorem 1, we can conclude:

Theorem 2 Assuming fair schedulings, $\forall k, 0 \leq k<4$, there is no protocol to (deterministically or probabilistically) explore any torus of $n$ nodes using $k$ robots.

Consider now the deterministic exploration with $k=4$ robots. Assume any $(\ell, L)$-Torus such that $\ell=L$ and $\ell$ is even. Then, it is possible to initially place the four robots in such way that they have all identical views and all their possible destinations looked identical (just form a square whose adjacent sides have length $\frac{\ell}{2}$ ). In this case, the adversary can choose to synchronously activate all robots at each step in such way that the initial symmetry continues: we obtain a fair execution which does not terminate. Hence, following the idea of [16], we have:

Theorem 3 Assuming fair schedulings, $\forall k, 0 \leq k \leq 4$, there is no protocol to deterministically explore all tori of $n$ nodes using $k$ robots.

Notice that the previous impossibility result can be circumvented, for example, by making restrictions on possible initial configurations [10]. 


\section{Optimal Algorithm}

We propose in Algorithm 1 a probabilistic protocol to explore with $\boldsymbol{k}=\mathbf{4}$ robots any $(\ell, L)$-torus such that $7 \leq \ell \leq L$, assuming weak multiplicity detection.

\subsection{Definitions}

We call a block a maximal elementary path along some ring of the torus $B=u_{i}, u_{i+1}, \ldots, u_{i+m}$ with $m>0$, where each node is occupied by exactly one robot. In $B$, the nodes $u_{i}$ and $u_{i+m}$ are termed as the extremities of $B$. The size of a block is the number of robots it contains. We call $x$-block any block of size $x$. A robot that does not belong to any block is said to be isolated.

We call hole any maximal non-empty elementary path of free nodes that is along some ring of the torus. The size of a hole is the number of nodes that it contains. We call $x$-hole any hole of size $x$. In the hole $H=u_{i}, \ldots, u_{k}(k \geq i)$ the nodes $u_{i}$ and $u_{k}$ are termed as the extremities of $H$. We call neighbor of a hole any node that does not belong to the hole but is neighbor of one of its nodes. In this case, we also say that the hole is a neighboring hole of the node. By extension, any robot that is located at a neighboring node of a hole is also referred to as a neighbor of the hole.

A node is safe if there is at most one robot that is located within distance one from it. We call Couple any $\ell$-ring that contains exactly two robots.

\subsection{Overview of the algorithm}

Our algorithm works in three distinct successive phases: Phase SetUp, Phase Tower, and Phase Exploration.

Starting from any towerless configuration, the aim of the Phase SetUp is to arrange the robots in such a way that they eventually form a $\diamond$.Configuration, without creating any tower during the process. This phase is probabilistic. A $\diamond$.Configuration is any configuration, where (1) there are two $\ell$-rings of the torus that both contain a 2-block, and (2) there are two robots that have two robots in their neighborhood. An example of a $\diamond$.Configuration is given in Figure 5.

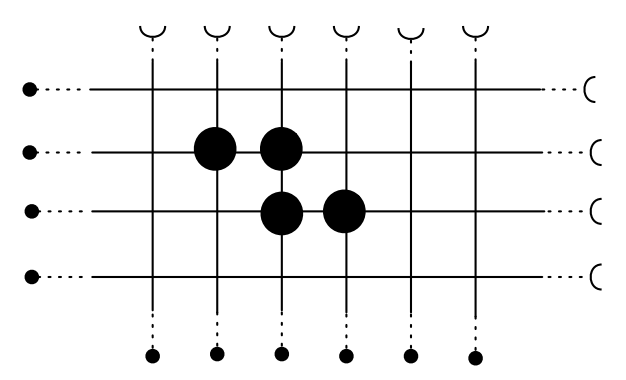

Figure 5: A $\diamond$.Configuration.

Once a $\diamond$.Configuration is reached, Phase Tower begins. This phase is also probabilistic and consists in creating a tower using the two neighboring robots that have exactly two robots in their neighborhood. Once the tower is created, the location of robots give an explicit orientation to the torus; and the last phase, Phase Exploration, begins. This phase is deterministic. The two isolated robots collaborate together to deterministically explore the torus and eventually stop.

Below, we detail each phase of our algorithm. 


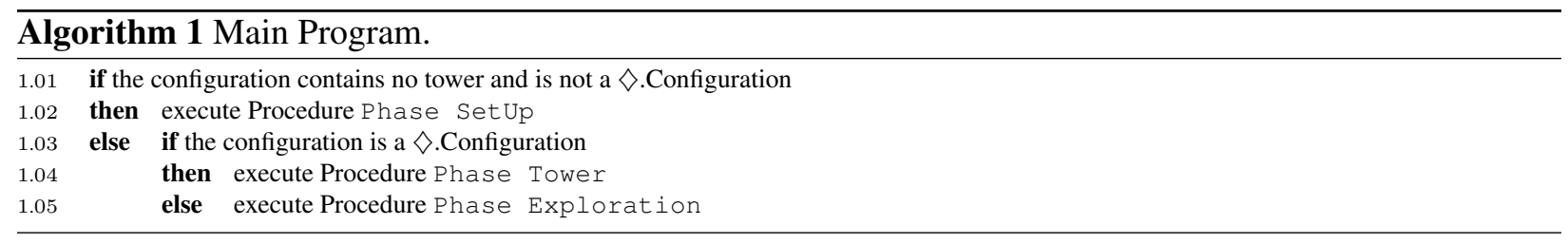

\subsection{Phase SetUp}

Phase SetUp is formally described in Algorithms 2-7.

\subsubsection{Configurations}

The behavior of Phase SetUp is led by the configurations. Below, we define the main classes of configurations.

A configuration is called Double-Trapl (refer to Figure 6) iff there exists a $\ell$-ring $R$ that contains a 3-block having exactly one extremity with a neighboring robot that is not in $R$.

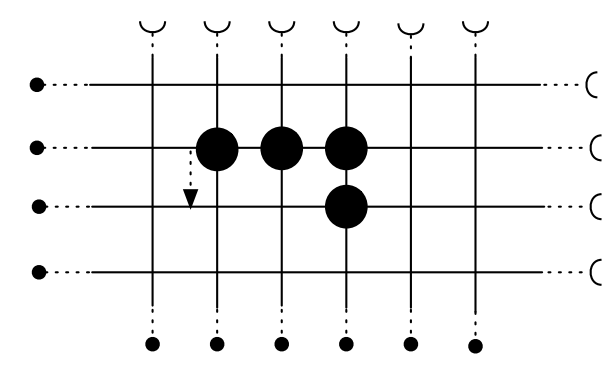

Figure 6: Double-Trapl Configuration.

A configuration is called Double-Trap2 (refer to Figure 7) iff there is one isolated robot at some node $z$ and two 2-blocks $B 1=u, v$ and $B 2=x, y$ such that (1) $v=x$, (2) $B 1$ is on a $\ell$-ring, (3) $z$ and $y$ are on a $\ell$-ring parallel to the one containing $B 1$, and (4) $z$ is at distance 2 of both $u$ and $y$.

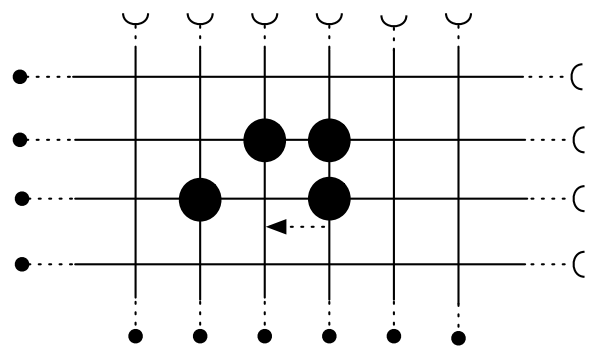

Figure 7: Double-Trap2 Configuration.

A configuration is called Isolated iff there exists at most one robot on each ring of size $\ell$. A configuration is called Triplet iff it is not a Double-Trap 1 configuration and there is a $\ell$-ring $R$ that contains 3 robots. When $R$ contains neither a 3-block nor a 2-block, we define the Wall as the ring perpendicular to $R$ that contains at most one or two robots (refer to Figure 8). 


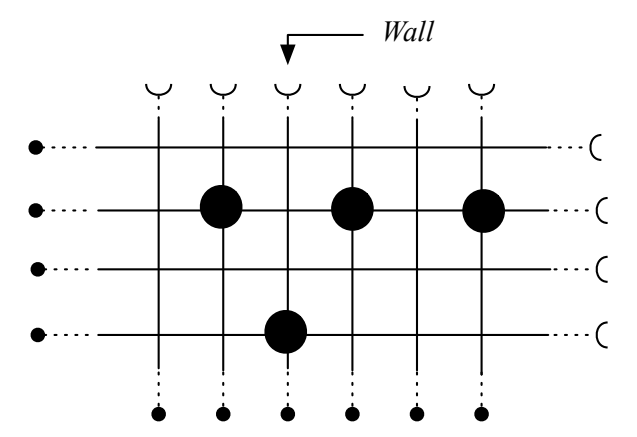

Figure 8: A wall in a Triplet Configuration.

A configuration is called Twin (refer to Figure 9) iff it contains a couple but it is neither a Double-Trap2 nor a $\diamond$.configuration.

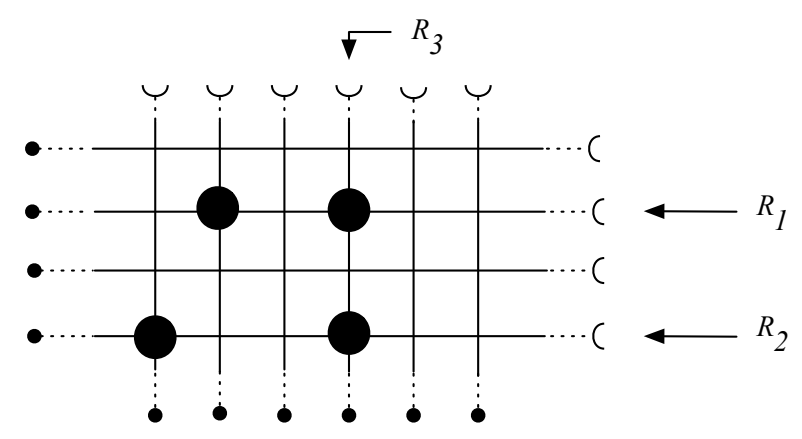

Figure 9: Twin Configuration

A configuration is called Quadruplet iff there exists a $\ell$-ring $R$ that contains 4 robots.

\subsubsection{Description of Phase SetUp}

Recall that the aim of the SetUp phase is to create a $\diamond$.Configuration, starting from an arbitrary towerless configuration and without creating any tower during the phase. As a matter fact, we try to make robots move deterministically as much as possible. However, there are symmetric configurations that require robots to move probabilistically. The main one is the following:

(a) The configuration is of type Identical. That is, all robots have identical views. To break this symmetry, each robot tries to move to a safe node. Doing so, the symmetry is broken after one step with positive probability.

So, with probability one (or almost surely, see [15], page 186), the system eventually leaves Case (a) to a configuration that matches one of the following cases:

(b) The configuration is of type Double-Trap2. In this case, only one robot moves as shown in Figure 7, we obtain a $\diamond$.Configuration, and the SetUp phase is done.

(c) The configuration is of type Double-Trapl. In this case, only one robot moves as shown in Figure 6 and we obtain a Double-Trap2 configuration. After one more move, we obtain a $\diamond$.Configuration and the SetUp phase is done. 
(d) The configuration is of type Triplet. In this case, there are three robots in the same $\ell$-ring $R$ and we deterministically build a Double-Trap1 configuration.

If three robots on $R$ already forms a 3-block, the remaining robot (not part of $R$ ) moves to the adequate position to build a Double-Trap1 configuration.

Otherwise, if $R$ contains a 2-block, then the robot in $R$ that is not part of the 2-block moves to create the 3-block. Otherwise, a Wall is defined and we use it to create a 2- or 3-block on $R$ : if the node $v$ that both intersects the Wall and $R$ is occupied by some robot, the two other robots on $R$ move towards the Wall to create a 2- or a 3-block (depending on the choices of the scheduler); otherwise, each robot of $R$ that is not neighbor of $v$ moves towards $v$ until a 2-block is created on $R$.

(e) The configuration is of type Twin. In this case, the aim is to reach a Triplet configuration with positive probability.

If the configuration contains only one couple, the two robots that are not part of the couple compete together (using try to move) so that eventually one of them is closer from the couple than the other. Then, the closest one moves to create the triplet configuration.

Now, the configuration may contain two or three couples (but not four, otherwise we would be on case (a)). In such cases, we consider the smallest rectangle, $S E R$, that encloses the 4 robots. If there are robots that are located at a corner of $S E R$ and belong to only one couple, such robots try to move to a neighboring safe node outside their couple. With a positive probability, only one of them moves and we retrieve a twin configuration that contains only one couple. In the case where there is no robot at the corner, then the two robots that are part of different couple being the farthest from each other try to move to a safe node.

In all remaining configurations, the aim is to reach either a triplet or twin configuration.

(f) The configuration is of type Quadruplet. In such a configuration, the four robots belong to some $\ell$-ring $R$. We then consider the subgraph $G_{R}$ induced by the nodes of $R$. This graph is isomorphic to an elementary cycle. Now, as not all robots have identical views, we can discriminate either one unique robot or two robots (using the sizes of the holes in $G_{R}$ ). We let those robots move outside $R$. Hence, the system reaches either a twin or a triplet configuration.

(g) The configuration is of type Isolated. We consider two subcases:

$-\ell>L$. In this case, we try to discriminate the $L$-rings according to the number of robots on them. When some but not all robots are alone in their $L$-rings, they move along their $L$-ring to eventually form a triplet or a twin configuration with the blocked ones.

In the case where one $L$-ring $R$ contains the four robots, we proceed as in Case $(f)$.

In the case where either every $L$-ring contains at most one robot, or two $L$-rings contain 2 robots, we again consider the smallest rectangle, $S E R$, that encloses the 4 robots. We can then discriminate robots according to their place in $S E R$ : at a corner, on a side, or inside of $S E R$. This allows us to block some of them. The other ones move to create either a triplet or a twin configuration.

$-\ell=L$. Again, we consider the smallest rectangle, $S E R$, that encloses the 4 robots. We discriminate robots as previously. However, note that this case is simpler than the previous one because all rings have the same size $(\ell)$, while in the previous case we had to take care that robots gather on a "small" ring. 

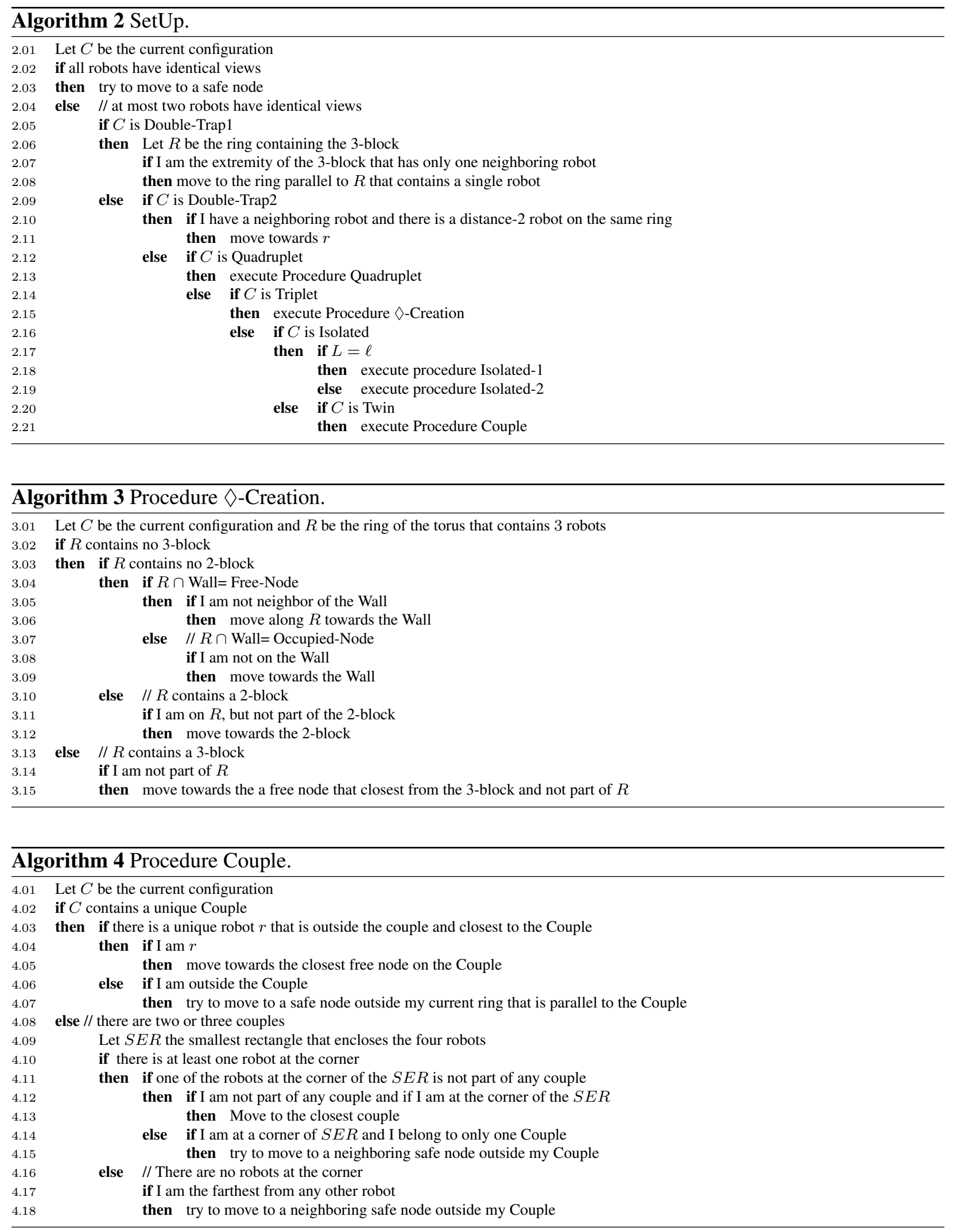

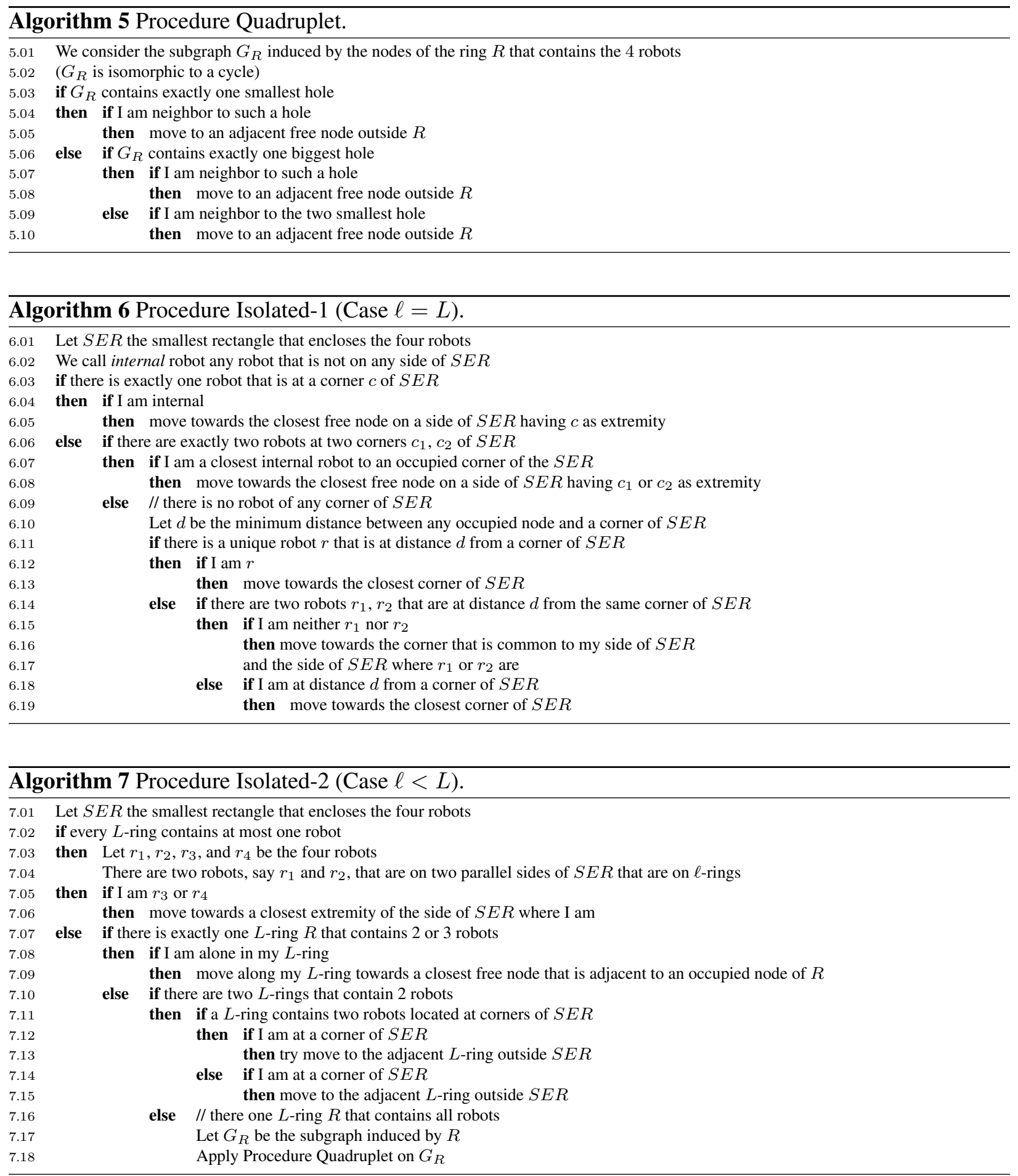

\subsection{Tower Phase}

This phase starts from any $\diamond$.Configuration, see for example Figure 5. Let $u_{1}$ and $u_{2}$ be the two occupied neighboring nodes having themselves two occupied neighboring nodes. Let $r_{1}$ and $r_{2}$ be the two robots located at $u_{1}$ and $u_{2}$, respectively. During this phase, $r_{1}$ and $r_{2}$ try to move towards each other anytime they are activated; the other two robots do not move if activated. If only one of them is activated, a tower 
is created. If both are activated simultaneously, there is a positive probability that only one of those moves; otherwise the system remains in a $\diamond$.Configuration. As the scheduler is fair, both of them are activated regularly until a tower is created. So, this latter event eventually occurs with probability one. Therefore, a tower $\mathrm{T}$ is created with probability one on either $u_{1}$ or $u_{2}-$ refer to Figure $10-$ and the Tower phase is finished.
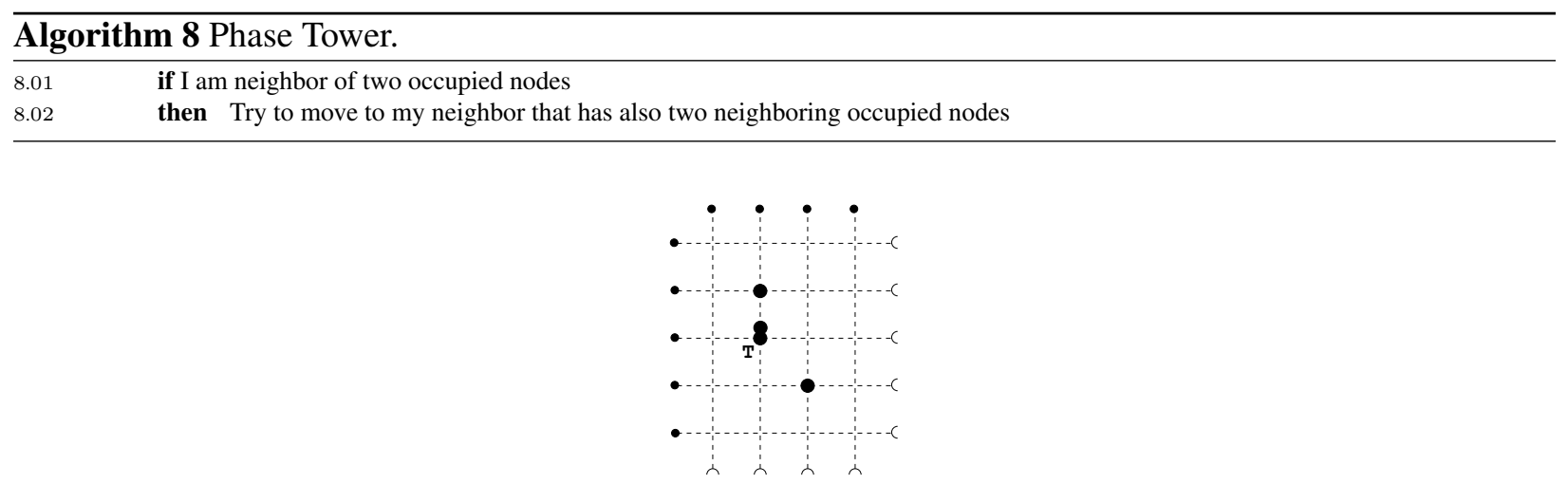

Figure 10: Initial Configuration for Deterministic Exploration.

\subsection{Phase Exploration}

We first need some definitions. Given two nodes $u$ and $v$, let $R_{u v}$ be the smallest enclosing rectangle that includes both $u$ and $v$, see Figure 11. Let $\alpha_{u v}\left(\beta_{u v}\right)$ be the length in terms of hops of one of the smallest (resp., greatest) side of $R_{u v}$, e.g., $\alpha_{u v}=2$ and $\beta_{u v}=4$ in Figure 11. $R_{u v}$ is an $\left(\alpha_{u v}, \beta_{u v}\right)$-rectangle. The (Manhattan) distance between two nodes $u$ and $v$, denoted by $d_{u v}$, is equal to $\alpha_{u v}+\beta_{u v}, e . g$., $d_{u v}=6$ in Figure 11. We define a total order on distances as follows: given four nodes $u, v, u^{\prime}$, and $v^{\prime}, d_{u v} \leq d_{u^{\prime} v^{\prime}}$ iff either $d_{u v}<d_{u^{\prime} v^{\prime}}$ or $d_{u v}=d_{u^{\prime} v^{\prime}}$ and $\beta_{u v} \leq \beta_{u^{\prime} v^{\prime}}$.

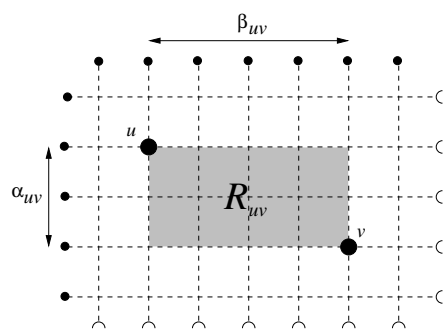

Figure 11: Smallest Enclosing Rectangle.

The deterministic exploration starts assuming that two robots form a tower. Denote the node holding the tower by $\mathrm{T}$. The two rings passing through $\mathrm{T}$ are called coordinate rings. In the sequel, 'o' (respectively, ' *') denotes the nearest (respectively, farthest) single node (or robot) from T, i.e., $d_{\circ T}<d_{* T}$. Note that our algorithm ensures that both ' $\circ$ ' and ' $*$ ' remain the same robots until the end of the exploration. Given a node $u$, if $\alpha_{T u}<\beta_{T u}$ and $\left\{\alpha_{T u}, \beta_{T u}\right\} \neq\left\{\left\lfloor\frac{\ell}{2}\right\rfloor,\left\lfloor\frac{L}{2}\right\rfloor\right\}$, then there exists an orientation of the coordinate rings such that $u=\left(\alpha_{T u}, \beta_{T u}\right)$, see for example Figure 12. In the following, when it is possible, we build a coordinate system over $R_{T u}$ by setting the $x$-axis (the $y$-axis) as the coordinate rings that is parallel to the smallest (resp., greatest) side of $R_{T, *}$ and by orienting both axis so that the coordinates of $u$ are positive.

The formal algorithm in shown in Algorithms 9 to 14. The main idea of Phase Exploration is the following: both robots that are not part of the tower collaborate together in order to explore the whole torus. They alternate between two roles: Explorer and Leader. Leader $L$ allows to build a coordinate system $S^{L}$ 


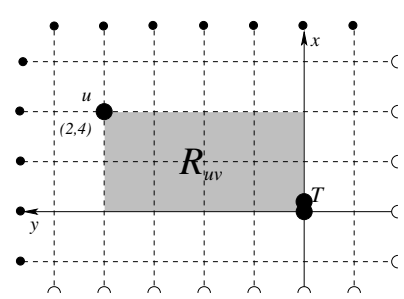

Figure 12: Coordinate System.

over $R_{\mathrm{T} L}$. The explorer is in charge of deterministically exploring the torus over $S^{\mathrm{L}}$. The exploration works in three phases, executed in sequence:

Phase 1: Figure 13 illustrates that phase. Robot $\star$ (i.e., the farthest robot of $\mathrm{T}$ ) plays the Leader role. Starting from the configuration built by Phase Tower, Robot $\star$ first built a $(1,2)$-rectangle with $\mathrm{T}$ by moving in the opposite direction to $\circ$, see Moving \#1 in Line 9.03. $R_{\mathrm{T} *}$ allows to build a coordinate system $S^{*}$, where Robot $\star$ occupies Node $(1,2)$ w.r.t. $S^{*}$. Then, Robot $\circ$ initiates a spiral-shaped exploration. It visits the nodes that form the first surrounding square around $\mathrm{T}$ and stops at node $(-1,-1)$, see Moving \#2 in Lines 9.04-9.10 and Procedure 1stSpiral(phase) in Algorithm 10.

Next, Robot $\star$ moves to node $(2,3)$ passing through node $(1,3)$, see Movings $3^{\prime}$ and $3^{\prime \prime}$ in Lines 9.08 and 9.14. Then, Robot $\circ$ visits the nodes that form the second surrounding square around $\mathrm{T}$ and stops at $(-2,-2)$, see Moving \#4 in Lines 9.26. Finally, Robot $\star$ moves back to $(1,3)$, followed by Robot $\circ$ that moves back to $(-2,-1)$, see Movings \#5 and \#6 in Lines 9.24 and 9.17. This ends the first phase.

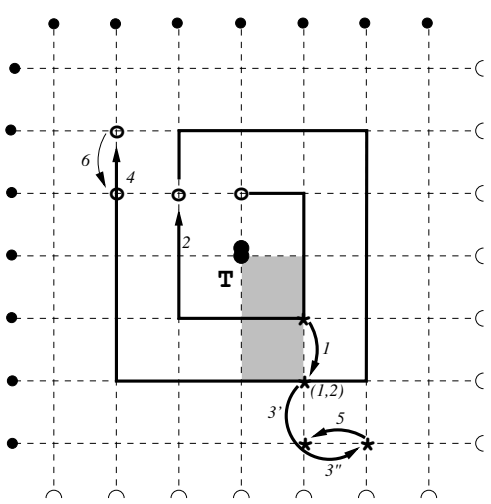

Figure 13: First phase of exploration.

Note that our method requires that Robot $\star$ must be able to move at least three lines away from the tower. Furthermore, Robot $\circ$ must be able to visit the two squares centered on the tower and the orientation built by Robot $\star$ must be unambiguous. These three conditions constrain the torus to be of size at least $7 \times 7$.

Phase 2: In this phase, Robot $\circ$ is the leader. $R_{\text {To }}$ provides a coordinate system $S^{\circ}$, where Robot $\circ$ is located at $(1,2)$. Robot $\star$ now proceeds to the spiral exploration by visiting surrounding squares around $\mathrm{T}$ one after another, see Figures 14 and 15. This process is initiated at Line 9.20 and managed by Procedures 2ndSpiralMngmt and 2ndSpiral (Algorithms 11 and 12). Robot $\star$ first explores the third surrounding square around $\mathrm{T}$, then the fourth, and so forth, until it visits the $\left(\left\lfloor\frac{\ell}{2}\right\rfloor-1\right)$-th square.

Then, there are two cases depending on the parity of $\ell$ : If $\ell$ is odd, then Robot $\star$ visits the whole $\left\lfloor\frac{\ell}{2}\right\rfloor$-th square and finish at the negative (w.r.t $S^{\circ}$ ) corner of the square, see for example Figure 14 . Otherwise $\left(\ell\right.$ is even), Robot $*$ visits half of the $\left\lfloor\frac{\ell}{2}\right\rfloor$-th square only and stops at the positive corner 
(w.r.t. $S^{\circ}$ ) of the square, see for example Figure 14. In both cases, if $\ell=L$, then the exploration is done, see Line 11.10.

Phase 3: This last phase is performed only if $\ell \neq L$. In that case, Robot $\star$ terminates the exploration by going alternatively from the left to the right and from the right to the left among the nodes forming the remaining of the rectangle. If $\ell$ is odd, then Robot $\star$ progresses towards the negative (w.r.t. $S^{\circ}$ ) side of the torus, see Figure 14 and Algorithm 13. Otherwise ( $\ell$ is even), the progression is made on the positive side, see Figure 15 and Algorithm 14. In both cases, the exploration ends either on the positive side or the negative side of the $L$-th line, depending on either $L$ is odd or even, see Figures 14-15 that shows two of the four possible cases of termination depending on the parity of both $\ell$ and $L$.

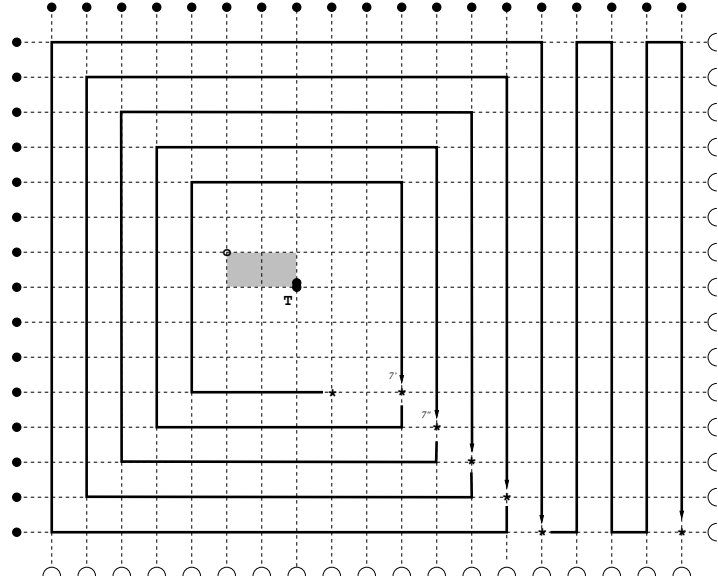

Figure 14: Second and third phase of exploration, odd case.

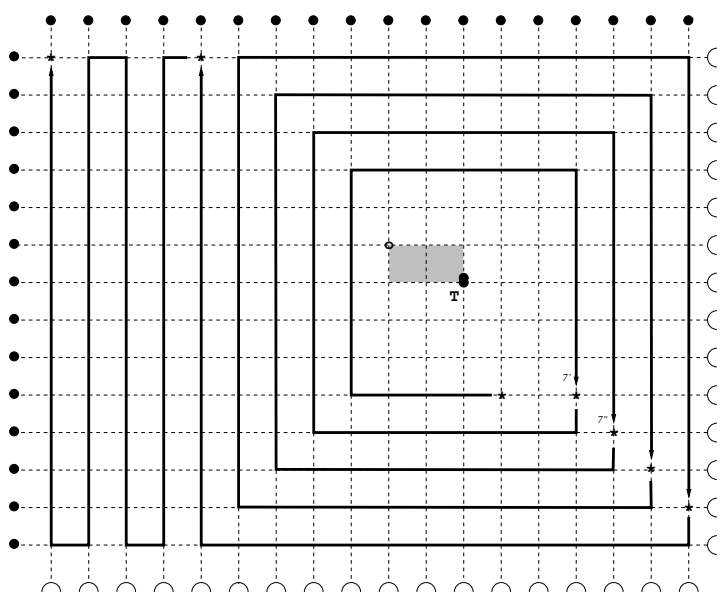

Figure 15: Second and third phase of exploration, even case.

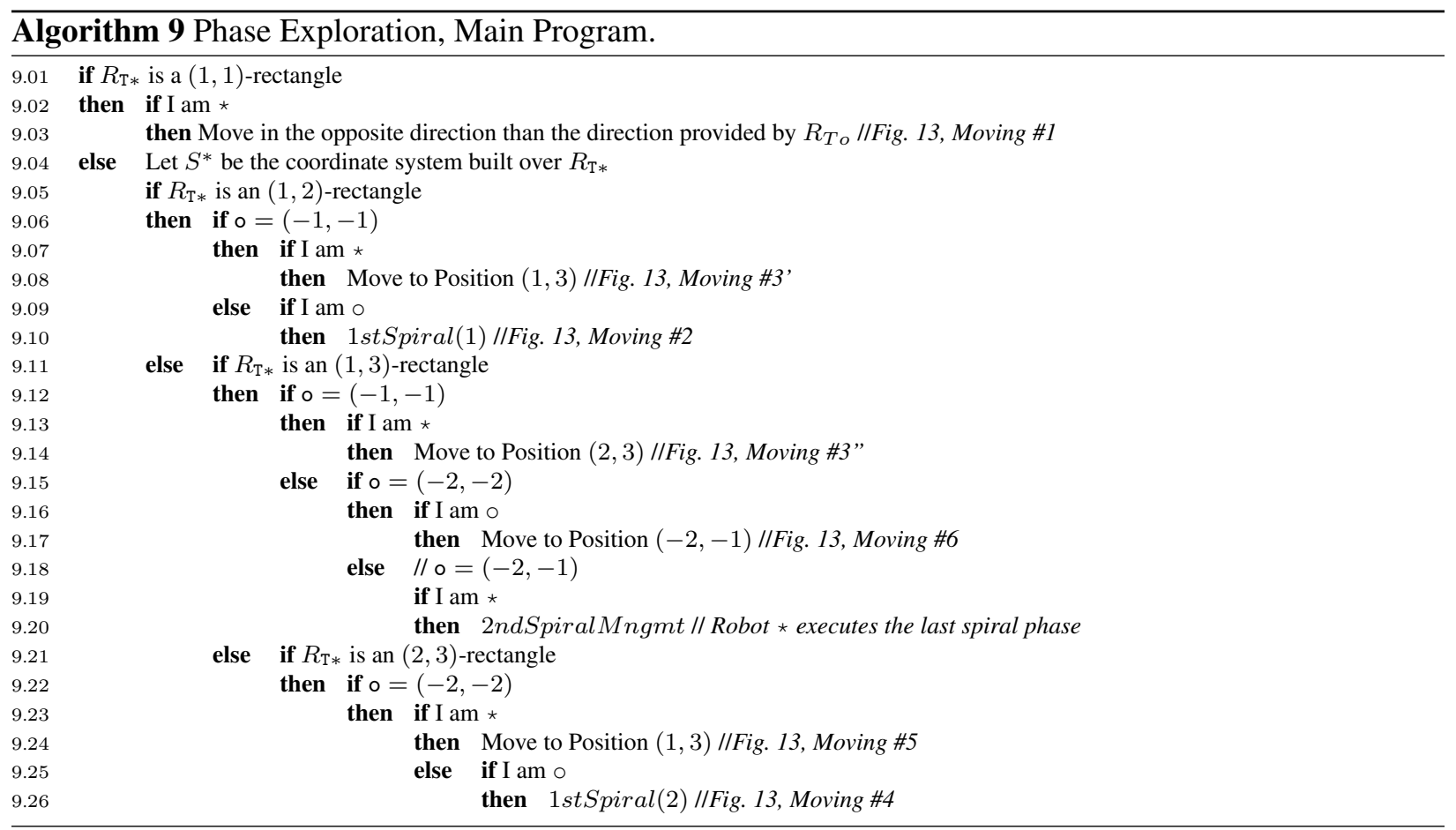



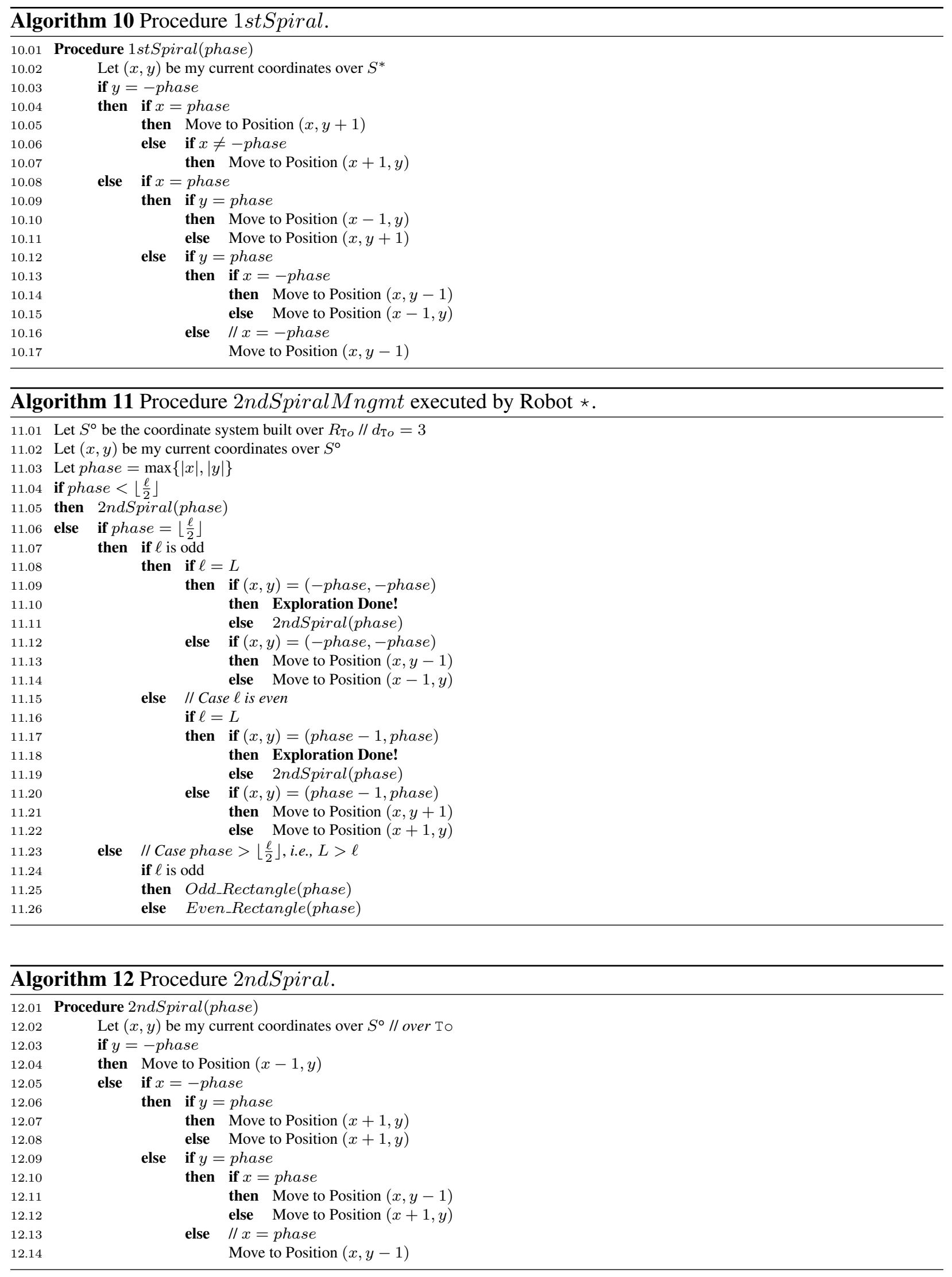

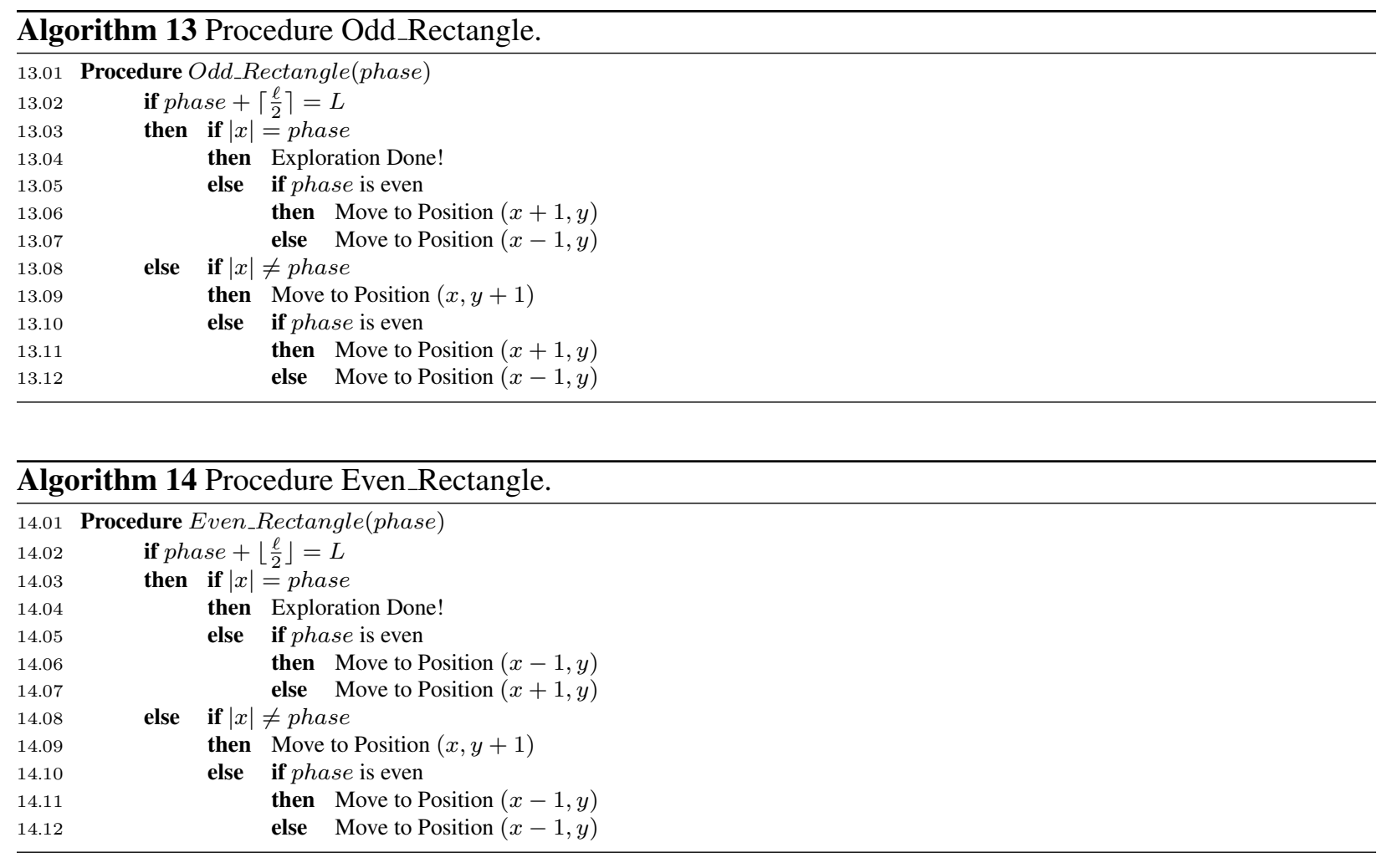

\section{Correctness Proof}

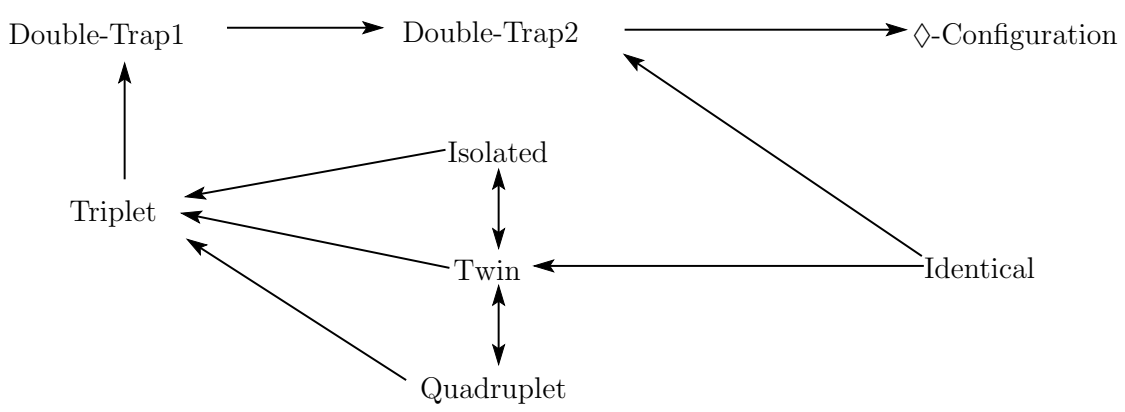

Figure 16: Possible transitions during SetUp.

Recall that Algorithm 1 is made of three phases: SetUp, Tower, and Exploration. The first phase, SetUp, starts from any towerless configuration and ends in a $\diamond$.Configuration. Moreover, this phase contains no configuration with a tower. (As a matter of fact, we show in Figure 16 the possible transitions between the different classes of configurations of SetUp; note that such transitions are either probabilistic or deterministic and may be performed in several steps.) The second phase, Tower, just consists in a single probabilistic transition (that may be performed in several steps) from a $\diamond$.Configuration to the initial towered configuration of the Exploration phase. Finally, all configurations that appear during the Exploration phase contain a tower. Hence, we have the following property:

Remark 3 The three phases of Algorithm 1 (SetUp, Tower, and Exploration) are unambiguous. 
Consider any $\diamond$.Configuration. Let $u_{1}$ and $u_{2}$ be the two occupied neighboring nodes having themselves two occupied neighboring nodes. Let $r_{1}$ and $r_{2}$ be the two robots located at $u_{1}$ and $u_{2}$, respectively. During this phase, $r_{1}$ and $r_{2}$ try to move towards each other anytime they are activated; the other two robots do not move if activated. If either $r_{1}$ or $r_{2}$, but not both, is activated, then a tower is created. If both $r_{1}$ and $r_{2}$ are activated simultaneously, then the following two events have positive probability to occur: either the system remains a $\diamond$.Configuration (because $r_{1}$ and $r_{2}$ both decide to either move or not move) or a tower is created (because either $r_{1}$ or $r_{2}$ decides to move). As the scheduler is fair, $r_{1}$ and $r_{2}$ are activated regularly until a tower is created, and with probability 1, the initial towered configuration of the Exploration phase is eventually reached.

Then, from the initial configuration of the Exploration phase, robots $\star$ and $\circ$ explore the torus in a fully deterministic manner. So, from such a configuration, the termination is guaranteed. Thus, we can conclude that starting from a $\diamond$.Configuration, the torus is eventually explored with probability one.

Hence, the critical part of the algorithm is the SetUp phase, whose aims at converging to a $\diamond$.Configuration starting from any initial (towerless) configuration. So, we now focus on this phase and show that, the convergence is obtained with probability one.

First, the SetUp phase exactly deals with the set of all towerless configurations. Now, by definition, we have:

Remark 4 The set of all towerless configurations is equal to the (disjoint) union of the following configuration sets:

1. $\diamond$.Configurations,

2. Identical,

3. Double-Trap1,

4. Double-Trap2,

5. Twin,

6. Triplet,

7. Quadruplet, and

8. Isolated.

The first part of the proof consists in showing that if the system is in a configuration of type DoubleTrap1, Double-Trap2 or Triplet, then it (deterministically) reaches a $\diamond$.Configuration in finite time.

In the following, we consider an arbitrary towerless configuration $C$.

Lemma 3 If $C$ is of type Double-Trap2, then a configuration $C^{\prime}$ of type $\diamond$.Configuration is reached from $C$ within finite time.

Proof. When the configuration $C$ is of type Double-Trap2, there is exactly one robot $\mathcal{R}$ that is allowed to move (refer to Algorithm 2, Line 8). $\mathcal{R}$ is the robot that has a neighboring robot and another robot at distance 2 on the same ring. Its destination is its adjacent free node towards the robot that is at distance 2 . As the scheduler is fair, $\mathcal{R}$ moves in finite time and then a configuration of type $\diamond$.Configuration is reached.

Lemma 4 If $C$ is of type Double-Trapl, then a configuration $C^{\prime}$ of type $\diamond$.Configuration is reached from $C$ within finite time.

Proof. When the configuration $C$ is of type Double-Trap1, the only robot allowed to move is the robot $\mathcal{R}$ that is at the extremity of the 3-block having exactly one neighbor (refer to Algorithm 2, Line 5). Its destination is its adjacent free node on the ring containing a single robot. As the scheduler is fair, $\mathcal{R}$ moves 
in finite time and a configuration of type Double-Trap2 is then reached. Hence, by Lemma 3, we are done.

Lemma 5 If $C$ is of type Triplet, then a configuration $C^{\prime}$ of type $\diamond$. Configuration is reached from $C$ within finite time.

Proof. Let $R$ be the ring that contains three robots in $C$. The cases below are possible:

1. $R$ contains a 3-block. In this case the robot that is not part of $R$ is the only one allowed to move (refer to Algorithm 3, Line 15). Its destination is its adjacent node on the shortest path towards the closest free node neighbor of one extremity of the 3-block (let us refer to this free node by $U$ ). Observe that this robot is the only one allowed to move unless it reaches $U$. Now, the scheduler being fair, the target node is reached in finite time and then a Double-Trap1 configuration is created. By Lemma 4, we are done in this case.

2. $R$ contains a 2-block. In this case, the robot that is on $R$, but not part of the 2-block is the only one allowed to move (refer to Algorithm 3, Line 11). Its destination is its adjacent free node on the shortest path towards the 2-block (on $R$ ). Note that this robot is the only one allowed to move unless it becomes part of a 3-block. Now, as the scheduler is fair, we retrieve Case 1 in finite time and we are done.

3. The other cases.

(a) If $R \cap$ wall= Occupied-Node. Then, the robots allowed to move are the two robots on $R$ that are not located at Occupied-Node (refer to Algorithm 3, Line 8). Their destination is their adjacent free node towards the wall. According to the choices of the fair scheduler, either a 3-block is created and we retrieve Case 1, or a 2-block is created and we retrieve Case 2.

(b) If $R \cap$ wall= Free-Node then, every robot of $R$ that is not neighbor of Free-Node moves towards Free-Node until a 2-block is created on $R$ (refer to Algorithm 3, Line 5). As the scheduler is fair, a 2-block is created on $R$ in finite time, and we retrieve Case 2.

From the other towerless configurations (Identical, Twin, Quadruplet, and Isolated), the convergence to a $\diamond$.Configuration is probabilistic. Now, when considering any arbitrary fair execution, (1) the topology is fixed with, in particular, a finite number of nodes and (2) recall that the number of robots is the constant 4. So, the number of towerless configurations is also finite. Now, while a configuration of type Double-Trap1, Double-Trap2, Triplet, or $\diamond$.Configuration is not reached, the SetUp phase remains trapped in the finite subset of towerless configurations defined as the distinct union of Configuration Identical, Twin, Quadruplet, and Isolated, by Remark 4. So, in order to demonstrate that the system eventually reaches a $\diamond$.Configuration with probability one, we show below that from any configuration of type Identical, Twin, Quadruplet, or Isolated, there always exists a positive probability to reach a configuration of type Double-Trap1, DoubleTrap2, or Triplet in finite time and despite the choices of the fair scheduler. (Remember that once such a configuration is reached, a $\diamond$.Configuration is reached within finite time, by Lemmas 3-5.)

Lemma 6 If $C$ is of type Twin then, with positive probability, a configuration of type Triplet is reached in finite time.

Proof. When the configuration $C$ is of type Twin, the cases bellow are possible ( $C$ cannot contains four couples, otherwise it would be of type Identical):

1. C contains a unique couple, $R$.

(a) If there is a unique robot $r$ that is outside $R$ and the closest to $R$, then $r$ is the only robot allowed to move, and if activated, it moves towards the closest free node in $R$ (refer to Lines from 4.03 to 4.05). Observe that by moving $r$ becomes even close to $R$. Thus, $r$ keeps being the only 
robot allowed to move until it becomes part of $R$. Hence, as the scheduler is fair, a Triplet configuration is reached, in this case, within finite time.

(b) In either cases, the two robots that are outside $R$ try to move to a safe node outside their current ring and that is parallel to $R$ (refer to Lines from 4.06 to 4.07 ). Then, as the scheduler is fair, at least one of them is activated in finite time, and when activated, with positive probability (actually, a probability greater or equal to $p(1-p)$ ), exactly one of those robots moves and we retrieve the previous case. Hence, we can conclude that, in this case, with positive probability, a configuration of type Triplet is reached in finite time.

2. C contains two couples. Two cases are possible:

(a) There is at least one robot at the corner of the $S E R$. ( $S E R$ is the smallest enclosing rectangle.) In this case, if one of the robots at the corner of the $S E R$ is not part of any Couple, then this robot is the only one allowed to move (refer to Algorithm 4, Lines 4.11 to 4.13). Its destination is its adjacent free node towards the closest couple. Then, as the scheduler is fair, it reaches a couple to form a Triplet configuration within finite time.

(b) Otherwise, the two robots at the corner of the SER try to move to a neighboring safe node (refer to Algorithm 4, Lines 4.14 to 4.15), if activated. As the scheduler is fair, at least one of them is activated within finite time and when activated, with positive probability (actually, a probability greater or equal to $p(1-p)$ ), exactly one of them moves. In this case, the system reaches either a Triplet or Case 1. Hence, with positive probability, a configuration of type Triplet is reached in finite time.

(c) There is no robot at the corner of the $S E R$. In this case, the torus necessarily satisfies $\ell=L$. Observe that, in this case, there exist two robots that are the farthest from each other, otherwise the configuration would be of type Identical. These robots try to move to a neighboring safe node, if activated (refer to Algorithm 4, Lines 4.16 to 4.18). As the scheduler is fair, at least one of them is activated in finite time, and when activated, with positive probability (actually, a probability greater or equal to $p(1-p)$ ), exactly one of them moves. In this case,

i. either, the configuration remains with two couples but this time there are robots on the corner. Thus, we retrieve Case $2 \mathrm{a}$, or

ii. a Triplet configuration is reached.

Hence, from the previous cases, we can conclude that, in this case, with positive probability, a configuration of type Triplet is reached in finite time.

3. $C$ contains three couples. (In this case, the torus necessarily satisfies $\ell=L$.) Observe that, in this case, there exist two robots that are part of two couples at the same time. The robots allowed to move are the other two robots. These robots try to move to a neighboring safe node. As the scheduler is fair, at least one of them is activated within finite time and when activated, with positive probability (actually, a probability greater or equal to $p(1-p)$ ), exactly one of them moves and the configuration reached is either Triplet or Twin but with only one or two couples, that is one of the previous cases. Hence, we can conclude that, in this case, with positive probability, a configuration of type Triplet is reached in finite time.

Lemma 7 If $C$ is of type Quadruplet then, a configuration $C^{\prime}$ of type either Twin or Triplet is reached from $C$ within finite time.

Proof. Let us refer to the ring that contains four robots in $C$ by $R$. We have the following possible cases:

- $R$ contains exactly one smallest hole. In this case, the two robots neighbor to such a hole move to an adjacent free node outside $R$, if activated (refer to Lines from 5.03 to 5.05). As the scheduler is fair, 
at least one of them is activated within finite time. Then, if both robots move simultaneously, then the reached configuration is of type Twin. If only one robot moves, then the reached configuration is of type Triplet.

- $R$ contains exactly one biggest hole. In this case too, the two robots neighbor to such a hole move to an adjacent free node outside $R$, if activated (refer to Lines from 5.06 to 5.08). As the scheduler is fair, at least one of them is activated within finite time. Then, if both robots move simultaneously, the reached configuration is of type Twin. If only one robot moves, then a Triplet configuration is reached.

- There is only one robot that is neighbor to the two smallest holes on $R$. This robot is the only one allowed to move. Its destination is its adjacent free node outside $R$ (refer to Lines from 5.09 to 5.10). As the scheduler is fair, this robot moves in finite time and then a configuration of type Triplet is reached.

From the cases above we can deduce that starting from $C$, either Twin or Triplet configuration is reached in finite time.

By Lemma 6, we have the following corollary from the previous lemma:

Corollary 2 If $C$ is of type Quadruplet then, with positive probability, a configuration of type Triplet is reached in finite time.

Lemma 8 If $C$ is of type Identical then, with positive probability, a configuration of type Triplet or DoubleTrap2 is reached in finite time.

Proof. If $C$ is of type Identical then every robot tries to move, if activated. Now, in this case, with positive probability, exactly one of them moves during the next step and a configuration of type either Double-Trap2 or Twin is then reached. In the latter case, we can conclude by Lemma 6.

Lemma 9 If $C$ is of type Isolated then, with positive probability, a configuration of type Triplet is reached in finite time.

Proof. We split the study into two main cases:

1. $\ell=L$. Let $S E R$ be the smallest rectangle that encloses the four robots, we call internal robot any robot that is not located on any side of $S E R$. The following subcases are possible:

(a) There is exactly one robot that is at a corner c of $S E R$. In this case there is exactly one internal robot. It is the only robot allowed to move, its destination is the closest free node on a side of $S E R$ having $c$ as extremity (refer to Lines from 6.03 to 6.05). Observe that this robot keep being enabled until it becomes on the same ring as $c$ (the $S E R$ is invariant). Thus, as the scheduler is fair, a Twin configuration is reached in finite time and by Lemma 6, we are done.

(b) There are exactly two robots at two corners $c_{1}$, $c_{2}$ of $S E R$. The other two robots are in this case internal (otherwise the configuration would contain a couple). The internal robots that are the closest to an occupied corner of the $S E R$ move towards the closest free node on a side of $S E R$ having $c_{1}$ or $c_{2}$ as an extremity (refer to Lines from 6.06 to 6.08). Note that in the case where there are two robots allowed to move, their target destination is different (recall that the configuration is of type Isolated). The robots that have moved keep moving towards the same target node until they become on the same ring as a robot on the corner of the $S E R$. Thus, as the scheduler is fair, a Twin configuration is reached in finite time and by Lemma 6 , we are done. 
(c) There is no robot at the corner of the $S E R$. In this case, observe that all robots are located at different sides of the $S E R$. Let $d$ be the minimum distance between any occupied node and a corner of $S E R$. The following subcases are then possible:

i. There is a unique robot $r$ that is at distance $d$ from a corner of $S E R$. $r$ is the only robot allowed to move. Its destination is its adjacent free node towards the closest corner (refer to Lines from 6.11 to 6.13). Observe that by moving $r$ becomes even close to the corner, thus $r$ keeps being the only one enabled to move until it becomes neighbor of the closest corner. Hence, as the scheduler is fair, we retrieve Case 1a within finite time, and we are done.

ii. There are two robots $r_{1}$ and $r_{2}$ that are at distance $d$ from a corner.

A. If $r_{1}$ and $r_{2}$ are closest to the same corner $c$, then the two other robots, say $r_{3}$ and $r_{4}$, move towards the corner that is common to their side of $S E R$ and the side of $S E R$ where either $r_{1}$ or $r_{2}$ are located, if activated (refer to Lines from 6.14 to 6.17). Note that since all robot are on the border of $S E R, r_{3}$ and $r_{4}$ have different targets. Thus, as the scheduler is fair, a Twin configuration is reached in finite time and by Lemma 6, we are done.

B. If $r_{1}$ and $r_{2}$ are closest to a corner of $S E R$, but not the same then, then $r_{1}$ and $r_{2}$ are the only robots allowed to move. Their destination is their adjacent free node towards their closest corner (refer to Lines from 6.18 to 6.19). As the scheduler is fair, in finite time, either their both reach their target simultaneously and we retrieve Case $1 \mathrm{~b}$, or we retrieve Case 1a. Hence, from the previous cases, we are done.

iii. There are three or four robots that are at distance $d$ from a corner of $S E R$. Observe that since that the configuration is not of type Identical, there is one or two corners that have only one robot at distance $d$ from them. Such a robot is enabled to move and its destination is its closest corner (refer to Lines from 6.18 to 6.19). As the scheduler is fair, in finite time, we retrieve either Case 1a or Case $1 \mathrm{~b}$ and we are done.

2. $\ell \neq L$. In this case, according to the number of robots on $L$-rings, we can distinguish the following cases:

(a) Every L-ring contains at most one robot. Every $L$-ring contains at most one robot. Let $r_{1}, r_{2}$, $r_{3}$, and $r_{4}$ be the four robots. Observe that there are two robots, say $r_{1}$ and $r_{2}$, that are on two parallel sides of $S E R$ that are on $\ell$-rings. The robots allowed to move are $r_{3}$ and $r_{4}$. They move towards a closest extremity of their side of $S E R$ (refer to Lines from 7.02 to 7.06). Note that by doing so, $r_{3}$ and $r_{4}$ keeps being allowed to move until they become on the same $\ell$-ring as either $r_{1}$ or $r_{2}$. Hence, as the scheduler is fair, Twin configuration is reached in finite time and by Lemma 6 , we are done.

(b) There is exactly one L-ring $R$ that contains 2 robots. The robots that are alone on their $L$-ring move along their $L$-ring towards a closest free node that is adjacent to $R$ (refer to Lines from 7.07 to 7.09). Observe that these robots keep the same target until one of them becomes on the same $\ell$-ring as another robot. Then, in finite time, the system reaches either a Triplet or a Twin configuration depending of the activations decided by the fair scheduler. In the latter case, with a positive probability, a Triplet configuration is then reached in finite time by Lemma 6 and we are done.

(c) There is exactly one L-ring $R$ that contains 3 robots. The case is similar to the previous one, refer to Lines from 7.07 to 7.09: in finite time, the system reaches a Twin configuration and by Lemma 6, we are done.

(d) There exist two L-rings that contain two robots. In this case, two cases are possible:

i. There is a L-ring that contains two robots located at corners of $S E R$. The robots that are at corners try to move, if activated, to their adjacent $L$-ring outside $S E R$ (note that there are only two robots at the corner of $S E R$, otherwise the configuration is Twin) (refer to Lines 
from 7.11 to 7.13). As the scheduler is fair, in finite time one or both of them are activated and with positive probability (actually, a probability greater or equal to $p(1-p)$ ), only one of them moves and we retrieve Case $2 b$.

ii. There are two robots at corners, say $r_{1}$ and $r_{2}$, that are part of different L-rings. $r_{1}$ and $r_{2}$ are the only robots allowed to move and their destination is their adjacent free node on the neighboring $L$-ring outside $S E R$ (refer to Lines from 7.14 to 7.15). As the scheduler is fair, in finite time, either $r_{1}, r_{2}$, or both are activated. In the two first cases, we retrieve Case $2 \mathrm{~b}$. In the latter case, we retrieve Case $2 \mathrm{a}$.

(e) There is one L-ring $R$ that contains all robots. Let $G R$ be the subgraph induced by $R$. The robots in this case apply Procedure Quadruplet on GR. By Corollary 2, we are done.

From all previous lemmas and corollary, we can deduce that, with probability one, the SetUp phase eventually terminates in a $\diamond$.Configuration. Hence, we can conclude with the following theorem:

Theorem 4 Assuming any fair scheduler, Algorithm 1 is a probabilistic exploration protocol for 4 robots on any torus of size $\ell \times L$, where $7 \leq \ell \leq L$.

\section{Concluding Remarks}

While the solution we provided for the torus exploration problem is optimal with respect to the number of robots involved in the exploration, there remain challenging open questions:

1. We presented an algorithm for all tori of size $\ell \times L$, where $7 \leq \ell \leq L$. Determining if our results can be extended to smaller tori is an interesting problem, as previous results [3] show e.g. that smaller grids require more robots. We expect mechanized approaches [17] to be valuable for investigating small size tori robot number requirements.

2. Going higher dimension (from a ring to a torus) did not increase the robot number complexity of the exploration problem. The issue of the $d$-dimensional tori (for $d>2$ ) remains open.

\section{References}

[1] Paola Flocchini, Giuseppe Prencipe, and Nicola Santoro. Distributed Computing by Oblivious Mobile Robots. Synthesis Lectures on Distributed Computing Theory. Morgan \& Claypool Publishers, 2012.

[2] J. Chalopin, P. Flocchini, B. Mans, and N. Santoro. Network exploration by silent and oblivious robots. In $W G$, pages 208-219, 2010.

[3] Stéphane Devismes, Anissa Lamani, Franck Petit, Pascal Raymond, and Sébastien Tixeuil. Optimal grid exploration by asynchronous oblivious robots. In SSS, pages 64-76, 2012.

[4] Stéphane Devismes, Franck Petit, and Sébastien Tixeuil. Optimal probabilistic ring exploration by semi-synchronous oblivious robots. Theor. Comput. Sci., 498:10-27, 2013.

[5] Paola Flocchini, David Ilcinkas, Andrzej Pelc, and Nicola Santoro. Remembering without memory: Tree exploration by asynchronous oblivious robots. Theor. Comput. Sci., 411(14-15):1583-1598, 2010.

[6] Paola Flocchini, David Ilcinkas, Andrzej Pelc, and Nicola Santoro. Computing without communicating: Ring exploration by asynchronous oblivious robots. Algorithmica, 65(3):562-583, 2013. 
[7] Roberto Baldoni, François Bonnet, Alessia Milani, and Michel Raynal. Anonymous graph exploration without collision by mobile robots. Inf. Process. Lett., 109(2):98-103, 2008.

[8] Roberto Baldoni, François Bonnet, Alessia Milani, and Michel Raynal. On the solvability of anonymous partial grids exploration by mobile robots. In OPODIS, pages 428-445, 2008.

[9] Gianlorenzo D’Angelo, Gabriele Di Stefano, Alfredo Navarra, Nicolas Nisse, and Karol Suchan. A unified approach for different tasks on rings in robot-based computing systems. In IPDPS Workshops, pages 667-676, 2013.

[10] Gianlorenzo D'Angelo, Alfredo Navarra, and Nicolas Nisse. Gathering and exclusive searching on rings under minimal assumptions. In ICDCN, pages 149-164, 2014.

[11] K. Haba, T. Izumi, Y. Katayama, N. Inuzuka, and K. Wada. On gathering problem in a ring for $2 \mathrm{n}$ autonomous mobile robots. In SSS, page Poster, 2008.

[12] Ralf Klasing, Adrian Kosowski, and Alfredo Navarra. Taking advantage of symmetries: Gathering of many asynchronous oblivious robots on a ring. Theor. Comput. Sci., 411(34-36):3235-3246, 2010.

[13] Anissa Lamani, Maria Potop-Butucaru, and Sébastien Tixeuil. Optimal deterministic ring exploration with oblivious asynchronous robots. In SIROCCO, pages 183-196, 2010.

[14] Ichiro Suzuki and Masafumi Yamashita. Distributed anonymous mobile robots: Formation of geometric patterns. SIAM J. Comput., 28(4):1347-1363, 1999.

[15] Daniel W. Stroock. Probability theory : an analytic view. Cambridge university press, Cambridge, New York, 1993.

[16] Paola Flocchini, David Ilcinkas, Andrzej Pelc, and Nicola Santoro. Computing without communicating: Ring exploration by asynchronous oblivious robots. In Proceedings of the International Conference on Principles of Distributed Systems (OPODIS), Lecture Notes in Computer Science (LNCS), pages 105-118. Springer Berlin / Heidelberg, December 2007.

[17] François Bonnet, Xavier Défago, Franck Petit, Maria Potop-Butucaru, and Sébastien Tixeuil. Brief announcement: Discovering and assessing fine-grained metrics in robot networks protocols. In SSS, pages 282-284, 2012. 